\title{
Strong and Weak Convergence Theorems for Common Solutions of Generalized Equilibrium Problems and Zeros of Maximal Monotone Operators
}

\author{
L.-C. Zeng, ${ }^{1,2}$ Q. H. Ansari, ${ }^{3}$ David S. Shyu, ${ }^{4}$ and J.-C. Yao ${ }^{5}$ \\ ${ }^{1}$ Department of Mathematics, Shanghai Normal University, Shanghai 200234, China \\ ${ }^{2}$ Scientific Computing Key Laboratory of Shanghai Universities, Shanghai 200234, China \\ ${ }^{3}$ Department of Mathematics, Aligarh Muslim University, Aligarh 202 002, India \\ ${ }^{4}$ Department of Finance, National Sun Yat-Sen University, Kaohsiung 80424, Taiwan \\ ${ }^{5}$ Department of Applied Mathematics, National Sun Yat-Sen University, Kaohsiung 80424, Taiwan \\ Correspondence should be addressed to J.-C. Yao, yaojc@math.nsysu.edu.tw
}

Received 27 October 2009; Accepted 12 January 2010

Academic Editor: Tomonari Suzuki

Copyright ( 2010 L.-C. Zeng et al. This is an open access article distributed under the Creative Commons Attribution License, which permits unrestricted use, distribution, and reproduction in any medium, provided the original work is properly cited.

The purpose of this paper is to introduce and study two modified hybrid proximal-point algorithms for finding a common element of the solution set EP of a generalized equilibrium problem and the set $T^{-1} 0 \cap \widetilde{T}^{-1} 0$ for two maximal monotone operators $T$ and $\widetilde{T}$ defined on a Banach space $X$. Strong and weak convergence theorems for these two modified hybrid proximal-point algorithms are established.

\section{Introduction}

Let $X$ be a real Banach space with its dual $X^{*}$. The mapping $J: X \rightarrow 2^{X^{*}}$ defined by

$$
J(x):=\left\{x^{*} \in X^{*}:\left\langle x^{*}, x\right\rangle=\|x\|^{2}=\left\|x^{*}\right\|^{2}\right\}, \quad \forall x \in X,
$$

is called the normalized duality mapping. From the Hahn-Banach theorem, it follows that $J(x) \neq \emptyset$ for each $x \in X$.

A Banach space $X$ is said to be strictly convex, if $\|x+y\| / 2<1$ for all $x, y \in U=\{z \in$ $X:\|z\|=1\}$ with $x \neq y$. $X$ is said to be uniformly convex if for each $\epsilon \in(0,2]$, there exists 
$\delta>0$ such that $\|x+y\| / 2 \leq 1-\delta$ for all $x, y \in U$ with $\|x-y\| \geq \epsilon$. Recall that each uniformly convex Banach space has the Kadec-Klee property, that is,

$$
\begin{gathered}
x_{n} \rightarrow x \\
\left\|x_{n}\right\| \longrightarrow\|x\|
\end{gathered} \Longrightarrow x_{n} \longrightarrow x
$$

It is well known that if $X^{*}$ is strictly convex, then $J$ is single-valued. In the sequel, we shall still denote the single-valued normalized duality mapping by $J$. Let $C$ be a nonempty closed convex subset of $X, f: C \times C \rightarrow \mathbb{R}$ a bifunction, and $A: C \rightarrow X^{*}$ a nonlinear mapping. Very recently, Zhang [1] considered and studied the generalized equilibrium problem of finding $\widehat{x} \in C$ such that

$$
f(\widehat{x}, y)+\langle A \widehat{x}, y-\widehat{x}\rangle \geq 0, \quad \forall y \in C .
$$

The set of solutions of (1.3) is denoted by EP. Problem (1.3) and related problems have been studied and investigated extensively in the literature; See, for example, [2-12] and references therein. Whenever $A \equiv 0$, problem (1.3) reduces to the equilibrium problem of finding $\widehat{x} \in C$ such that

$$
f(\widehat{x}, y) \geq 0, \quad \forall y \in C
$$

The set of solutions of (1.4) is denoted by $E P(f)$. Whenever $f \equiv 0$, problem (1.3) reduces to the variational inequality problem of finding $\widehat{x} \in C$ such that

$$
\langle A \widehat{x}, y-\widehat{x}\rangle \geq 0, \quad \forall y \in C
$$

The set of solutions of (1.5) is denoted by $V I(C, A)$.

Whenever $X=H$ a Hilbert space, problem (1.3) was very recently introduced and considered by S. Takahashi and W. Takahashi [13]. Problem (1.3) is very general in the sense that it includes, as spacial cases, optimization problems, variational inequalities, minimax problems, Nash equilibrium problem in noncooperative games, and others; See, for example, $[1,2,4,6-9,14-17]$ which are references therein.

A mapping $S: C \rightarrow X$ is called nonexpansive if $\|S x-S y\| \leq\|x-y\|$ for all $x, y \in C$. Denote by $F(S)$ the set of fixed points of $S$, that is, $F(S)=\{x \in C: S x=x\}$. Very recently, $\mathrm{W}$. Takahashi and K. Zembayashi [18] proposed an iterative algorithm for finding a common element of the solution set of the equilibrium problem (1.4) and the set of fixed points of a relatively nonexpansive mapping $S$ in a Banach space $X$. They also studied the strong and weak convergence of the sequences generated by their algorithm. In particular, they proposed the following iterative algorithm:

$$
\begin{gathered}
x_{0} \in C, \\
y_{n}=J^{-1}\left(\alpha_{n} J x_{n}+\left(1-\alpha_{n}\right) J S x_{n}\right),
\end{gathered}
$$




$$
\begin{gathered}
u_{n} \in C \text { such that } f\left(u_{n}, y\right)+\frac{1}{r_{n}}\left\langle y-u_{n}, J u_{n}-J y_{n}\right\rangle \geq 0, \quad \forall y \in C, \\
H_{n}=\left\{z \in C: \phi\left(z, u_{n}\right) \leq \phi\left(z, x_{n}\right)\right\}, \\
W_{n}=\left\{z \in C:\left\langle x_{n}-z, J x-J x_{n}\right\rangle \geq 0\right\}, \\
x_{n+1}=\prod_{H_{n} \cap W_{n}} x, \quad n \geq 0,
\end{gathered}
$$

where $\phi(x, y)=\|x\|^{2}-2\langle x, J y\rangle+\|y\|^{2}$ for all $x, y \in X,\left\{\alpha_{n}\right\} \subset[0,1]$, and $\left\{r_{n}\right\} \subset[a, \infty)$ for some $a>0$. They proved that the sequence $\left\{x_{n}\right\}$ generated by the above algorithm converges strongly to $\Pi_{F(S) \cap E P(f)} x_{0}$, where $\Pi_{F(S) \cap E P(f)}$ is the generalized projection of $X$ onto $F(S) \cap$ $E P(f)$. They have also studied the weak convergence of the sequence $\left\{x_{n}\right\}$ generated by the following algorithm:

$$
\begin{gathered}
u_{0} \in X, \\
x_{n} \in C \text { such that } f\left(x_{n}, y\right)+\frac{1}{r_{n}}\left\langle y-x_{n}, J x_{n}-J u_{n}\right\rangle \geq 0, \quad \forall y \in C, \\
u_{n+1}=J^{-1}\left(\alpha_{n} J x_{n}+\left(1-\alpha_{n}\right) J S x_{n}\right), \quad n \geq 0,
\end{gathered}
$$

to $z \in F(S) \cap E P(f)$, where $z=\lim _{n \rightarrow \infty} \Pi_{F(S) \cap E P(f)} x_{n}$.

Let $C$ be a nonempty closed convex subset of a uniformly smooth and uniformly convex Banach space $X$. Let $A: C \rightarrow X^{*}$ be an $\alpha$-inverse-strongly monotone mapping and $f: C \times C \rightarrow \mathbb{R}$ a bifunction satisfying the following conditions:

(A1) $f(x, x)=0$ for all $x \in C$;

(A2) $f$ is monotone, that is, $f(x, y)+f(y, x) \leq 0$, for all $x, y \in C$;

(A3) for all $x, y, z \in C, \lim \sup _{t \downarrow} f(t z+(1-t) x, y) \leq f(x, y)$;

(A4) for all $x \in C, f(x, \cdot)$ is convex and lower semicontinuous.

Let $S_{1}, S_{2}: C \rightarrow C$ be two relatively nonexpansive mappings such that $F\left(S_{1}\right) \cap F\left(S_{2}\right) \cap$ $E P \neq \emptyset$. Let $\left\{x_{n}\right\}$ be the sequence generated by

$$
\begin{gathered}
x_{0} \in C, \quad C_{0}=C ; \\
z_{n}=J^{-1}\left(\alpha_{n} J x_{n}+\left(1-\alpha_{n}\right) J S_{1} x_{n}\right), \\
y_{n}=J^{-1}\left(\beta_{n} J x_{n}+\left(1-\beta_{n}\right) J S_{2} z_{n}\right), \\
u_{n} \in C \text { such that } f\left(u_{n}, y\right)+\left\langle A u_{n}, y-u_{n}\right\rangle+\frac{1}{r_{n}}\left\langle y-u_{n}, J u_{n}-J y_{n}\right\rangle \geq 0, \quad \forall y \in C, \\
C_{n+1}=\left\{v \in C_{n}: \phi\left(v, u_{n}\right) \leq \beta_{n} \phi\left(v, x_{n}\right)+\left(1-\beta_{n}\right) \phi\left(v, z_{n}\right) \leq \phi\left(v, x_{n}\right)\right\} ; \\
x_{n+1}=\prod_{C_{n+1}} x_{0}, \quad \forall n \geq 0 .
\end{gathered}
$$

Zhang [1] proved the strong convergence of the sequence $\left\{x_{n}\right\}$ to $\prod_{F\left(S_{1}\right) \cap F\left(S_{2}\right) \cap E P} x_{0}$ under appropriate conditions. 
On the other hand, a classic method of solving $0 \in T x$ in a Hilbert space $H$ is the proximal point algorithm which generates, for any starting point $x_{0} \in H$, a sequence $\left\{x_{n}\right\}$ in $H$ by the iterative scheme

$$
x_{n+1}=J_{r_{n}} x_{n}, \quad n=0,1,2, \ldots,
$$

where $\left\{r_{n}\right\}$ is a sequence in $(0, \infty), J_{r}=(I+r T)^{-1}$ for each $r>0$ is the resolvent operator for $T$, and $I$ is the identity operator on $H$. This algorithm was first introduced by Martinet [19] and further studied by Rockafellar [20] in the framework of a Hilbert space $H$. Later several authors studied (1.9) and its variants in the setting of a Hilbert space $H$ or in a Banach space $X$; See, for example, $[15,21-25]$ and references therein. Very recently, Li and Song [24] introduced and studied the following iterative scheme:

$$
\begin{gathered}
x_{0} \in X \text { chosen arbitrarily, } \\
y_{n}=J^{-1}\left(\beta_{n} J x_{n}+\left(1-\beta_{n}\right) J J_{r_{n}} x_{n}\right), \\
x_{n+1}=J^{-1}\left(\alpha_{n} J x_{0}+\left(1-\alpha_{n}\right) J y_{n}\right), \quad n=0,1,2, \ldots,
\end{gathered}
$$

where $J_{r}=(J+r T)^{-1} J$ and $J$ is the duality mapping on $X$.

Algorithm (1.10) covers, as special cases, the algorithms introduced by Kohsaka and Takahashi [23] and Kamimura et al. [22] in a smooth and uniformly convex Banach space X.

Let $X$ be a uniformly smooth and uniformly convex Banach space, and let $C$ be a nonempty closed convex subset of $X$. Let $T: X \rightarrow 2^{X^{*}}$ be a maximal monotone operator such that:

(A5) $T^{-1} 0 \cap E P(f) \neq \emptyset$.

In addition, for each $r>0$, define a mapping $T_{r}: X \rightarrow C$ as follows:

$$
T_{r}(x)=\left\{z \in C: f(z, y)+\frac{1}{r}\langle y-z, J z-J x\rangle \geq 0, \forall y \in C\right\}
$$

for all $x \in X$.

Very recently, utilizing the ideas of the above algorithms in $[15,16,18,21,22,24]$, we [17] introduced two iterative methods for finding an element of $T^{-1} 0 \cap E P(f)$ and established the following strong and weak convergence theorems.

Theorem 1.1 (see [17]). Suppose that conditions (A1)-(A5) are satisfied and let $x_{0} \in X$ be chosen arbitrarily. Consider the sequence

$$
x_{n+1}=\Pi_{H_{n} \cap W_{n}} x_{0}, \quad n=0,1,2, \ldots
$$


where

$$
\begin{gathered}
H_{n}=\left\{z \in C: \phi\left(z, T_{r_{n}} y_{n}\right) \leq \alpha_{n} \phi\left(z, x_{0}\right)+\left(1-\alpha_{n}\right) \phi\left(z, x_{n}\right)\right\}, \\
W_{n}=\left\{z \in C:\left\langle x_{n}-z, J x_{0}-J x_{n}\right\rangle \geq 0\right\} \\
y_{n}=J^{-1}\left(\alpha_{n} J x_{0}+\left(1-\alpha_{n}\right)\left(\beta_{n} J x_{n}+\left(1-\beta_{n}\right) J J_{r_{n}} x_{n}\right)\right)
\end{gathered}
$$

$T_{r}$ is defined by (1.11), $\left\{\alpha_{n}\right\},\left\{\beta_{n}\right\} \subset[0,1]$ satisfy $\lim _{n \rightarrow \infty} \alpha_{n}=0, \liminf _{n \rightarrow \infty} \beta_{n}\left(1-\beta_{n}\right)>0$, and $\left\{r_{n}\right\} \subset(0, \infty)$ satisfies $\liminf _{n \rightarrow \infty} r_{n}>0$. Then, the sequence $\left\{x_{n}\right\}$ converges strongly to $\Pi_{T^{-1} O \cap E P(f)} x_{0}$, where $\prod_{T^{-1} 0 \cap E P(f)}$ is the generalized projection of $X$ onto $T^{-1} 0 \cap E P(f)$.

Theorem 1.2 (see [17]). Suppose that conditions (A1)-(A5) are satisfied and let $x_{0} \in X$ be chosen arbitrarily. Consider the sequence

$$
x_{n+1}=J^{-1}\left(\alpha_{n} J x_{0}+\left(1-\alpha_{n}\right)\left(\beta_{n} J T_{r_{n}} x_{n}+\left(1-\beta_{n}\right) J J_{r_{n}} T_{r_{n}} x_{n}\right)\right), \quad n=0,1,2, \ldots,
$$

where $T_{r}$ is defined by (1.11), $\left\{\alpha_{n}\right\},\left\{\beta_{n}\right\} \subset[0,1]$ satisfy the conditions $\sum_{n=0}^{\infty} \alpha_{n}<\infty$ and $\liminf _{n \rightarrow \infty} \beta_{n}\left(1-\beta_{n}\right)>0$, and $\left\{r_{n}\right\} \subset(0, \infty)$ satisfies $\liminf _{n \rightarrow \infty} r_{n}>0$. If $J$ is weakly sequentially continuous, then $\left\{x_{n}\right\}$ converges weakly to an element $z \in T^{-1} 0 \cap \operatorname{EP}(f)$, where $z=\lim _{n \rightarrow \infty} \Pi_{T^{-1} 0 \cap E P(f)} x_{n}$.

The purpose of this paper is to introduce and study two new iterative methods for finding a common element of the solution set $E P$ of generalized equilibrium problem (1.3) and the set $T^{-1} 0 \cap \widetilde{T}^{-1} 0$ for maximal monotone operators $T$ and $\widetilde{T}$ in a uniformly smooth and uniformly convex Banach space X. Firstly, motivated by Theorem 1.1 and a result of Zhang [1], we introduce a sequence $\left\{x_{n}\right\}$ that converges strongly to $\Pi_{T^{-1} 0 \cap \tilde{T}^{-1} 0 \cap E P} x_{0}$ under some appropriate conditions.

Secondly, inspired by Theorem 1.2 and a result of Zhang [1], we define a sequence that converges weakly to an element $z \in T^{-1} 0 \cap \widetilde{T}^{-1} 0 \cap E P$, where $z=\lim _{n \rightarrow \infty} \Pi_{T^{-1} 0 \cap \tilde{T}^{-1} 0 \cap E P} x_{n}$ (Section 4).

Our results represent a generalization of known results in the literature, including those in $[16-18,24]$. Our Theorems 3.1 and 4.2 are the extension and improvements of Theorems 1.1 and 1.2 in the following way:

(i) the problem of finding an element of $T^{-1} 0 \cap \widetilde{T}^{-1} 0 \cap E P$ includes the one of finding an element of $T^{-1} 0 \cap E P(f)$ as a special case;

(ii) the algorithms in this paper are very different from those in [17] because of considering the complexity involving the problem of finding an element of $T^{-1} 0 \cap$ $\widetilde{T}^{-1} 0 \cap E P$.

\section{Preliminaries}

Throughout the paper, we denote the strong convergence, weak convergence, and weak ${ }^{*}$ convergence of a sequence $\left\{x_{n}\right\}$ to a point $x \in X$ by $x_{n} \rightarrow x, x_{n} \rightarrow x$ and $x_{n} \stackrel{*}{\rightarrow} x$, respectively.

Assumption 2.1. Let $X$ be a uniformly smooth and uniformly convex Banach space and let $C$ be a nonempty closed convex subset of $X$. Let $A: C \rightarrow X^{*}$ be an $\alpha$-inverse-strongly monotone 
mapping and let $f: C \times C \rightarrow \mathbb{R}$ be a bifunction satisfying the conditions (A1)-(A4). Let $T, \tilde{T}: X \rightarrow 2^{X^{*}}$ be two maximal monotone operators such that:

$$
(\mathrm{A} 5)^{\prime} T^{-1} 0 \cap \tilde{T}^{-1} 0 \cap E P \neq \emptyset .
$$

Recall that if $C$ is a nonempty closed convex subset of a Hilbert space $H$, then the metric projection $P_{C}: H \rightarrow C$ of $H$ onto $C$ is nonexpansive. This fact actually characterizes Hilbert spaces and hence, it is not available in more general Banach spaces. In this connection, Alber [26] recently introduced a generalized projection operator $\Pi_{C}$ in a Banach space $X$ which is an analogue of the metric projection in Hilbert spaces.

Consider the functional defined as in [26] by

$$
\phi(x, y)=\|x\|^{2}-2\langle x, J y\rangle+\|y\|^{2}, \quad \forall x, y \in X
$$

It is clear that in a Hilbert space $H,(2.1)$ reduces to $\phi(x, y)=\|x-y\|^{2}, \forall x, y \in H$.

The generalized projection $\Pi_{C}: X \rightarrow C$ is a mapping that assigns to an arbitrary point $x \in X$ the minimum point of the functional $\phi(y, x)$, that is, $\Pi_{C} x=\bar{x}$, where $\bar{x}$ is the solution to the minimization problem

$$
\phi(\bar{x}, x)=\min _{y \in C} \phi(y, x)
$$

The existence and uniqueness of the operator $\Pi_{C}$ follow from the properties of the functional $\phi(x, y)$ and strict monotonicity of the mapping $J$; See, for example, [27]. In a Hilbert space, $\Pi_{C}=P_{C}$. From [26], in a smooth, strictly convex and reflexive Banach space $X$, we have

$$
(\|y\|-\|x\|)^{2} \leq \phi(y, x) \leq(\|y\|+\|x\|)^{2}, \quad \forall x, y \in X
$$

Moreover, by the property of subdifferential of convex functions, we easily get the following inequality:

$$
\phi(x, y) \leq \phi\left(x, J^{-1}(J y+J z)\right)-2\langle y-x, J z\rangle, \quad \forall x, y, z \in X
$$

Let $S$ be a mapping from $C$ into itself. A point $p$ in $C$ is called an asymptotic fixed point of $S$ [28] if $C$ contains a sequence $\left\{x_{n}\right\}$ which converges weakly to $p$ such that $\left\|S x_{n}-x_{n}\right\| \rightarrow 0$. The set of asymptotic fixed points of $S$ is denoted by $\widehat{F}(S)$. A mapping $S$ from $S$ into itself is called relatively nonexpansive $[18,29,30]$ if $\widehat{F}(S)=F(S)$ and $\phi(p, S x) \leq \phi(p, x)$, for all $x \in C$ and $p \in F(S)$.

Observe that, if $X$ is a reflexive, strictly convex and smooth Banach space, then for any $x, y \in X, \phi(x, y)=0$ if and only if $x=y$. To this end, it is sufficient to show that if $\phi(x, y)=0$, then $x=y$. Actually, from (2.3), we have $\|x\|=\|y\|$, which implies that $\langle x, J y\rangle=\|x\|^{2}=\|y\|^{2}$. From the definition of $J$, we have $J x=J y$ and therefore, $x=y$. For further details, we refer to [31].

We need the following lemmas for the proof of our main results. 
Lemma 2.2 (see [32]). Let $X$ be a smooth and uniformly convex Banach space and let $\left\{x_{n}\right\}$ and $\left\{y_{n}\right\}$ be two sequences of $X$. If $\phi\left(x_{n}, y_{n}\right) \rightarrow 0$ and either $\left\{x_{n}\right\}$ or $\left\{y_{n}\right\}$ is bounded, then $\left\|x_{n}-y_{n}\right\| \rightarrow 0$.

Lemma 2.3 (see $[26,32]$ ). Let $C$ be a nonempty closed convex subset of a smooth, strictly convex and reflexive Banach space $X, x \in X$ and $z \in C$. Then

$$
z=\Pi_{C} x \Longleftrightarrow\langle y-z, J x-J z\rangle \leq 0, \quad \forall y \in C
$$

Lemma 2.4 (see $[26,32]$ ). Let $C$ be a nonempty closed convex subset of a smooth, strictly convex and reflexive Banach space $X$. Then

$$
\phi\left(x, \Pi_{C} y\right)+\phi\left(\Pi_{C} y, y\right) \leq \phi(x, y), \quad \forall x \in C, y \in X
$$

Lemma 2.5 (see [33]). Let $X$ be a reflexive, strictly convex and smooth Banach space and let $T$ : $X \rightarrow 2^{X^{*}}$ be a multivalued operator. Then

(i) $T^{-1} 0$ is closed and convex if $T$ is maximal monotone such that $T^{-1} 0 \neq \emptyset$;

(ii) $T$ is maximal monotone if and only if $T$ is monotone with $R(J+r T)=X^{*}$ for all $r>0$.

Lemma 2.6 (see [34]). Let $X$ be a uniformly convex Banach space and let $r>0$. Then there exists a strictly increasing, continuous and convex function $g:[0,2 r] \rightarrow \mathbb{R}$ such that $g(0)=0$ and

$$
\|t x+(1-t) y\|^{2} \leq t\|x\|^{2}+(1-t)\|y\|^{2}-t(1-t) g(\|x-y\|),
$$

for all $x, y \in B_{r}$ and $t \in[0,1]$, where $B_{r}=\{z \in X:\|z\| \leq r\}$.

Lemma 2.7 (see [32]). Let $X$ be a smooth and uniformly convex Banach space and let $r>0$. Then there exists a strictly increasing, continuous, and convex function $g:[0,2 r] \rightarrow \mathbb{R}$ such that $g(0)=0$ and

$$
g(\|x-y\|) \leq \phi(x, y), \quad \forall x, y \in B_{r} .
$$

The following result is due to Blum and Oettli [14].

Lemma 2.8 (see [14]). Let $C$ be a nonempty closed convex subset of a smooth, strictly convex and reflexive Banach space $X, f: C \times C \rightarrow \mathbb{R}$ a bifunction satisfying conditions (A1)-(A4), and $r>0$ and $x \in X$. Then, there exists $z \in C$ such that

$$
f(z, y)+\frac{1}{r}\langle y-z, J z-J x\rangle \geq 0, \quad \forall y \in C .
$$

Motivated by a result in [35] in a Hilbert space setting, Takahashi and Zembayashi [18] established the following lemma. 
Lemma 2.9 (see [18]). Let $C$ be a nonempty closed convex subset of a uniformly smooth, strictly convex and reflexive Banach space $X$, and $f: C \times C \rightarrow \mathbb{R}$ a bifunction satisfying conditions (A1)(A4). For $r>0$ and $x \in X$, define a mapping $T_{r}: X \rightarrow C$ as follows:

$$
T_{r}(x)=\left\{z \in C: f(z, y)+\frac{1}{r}\langle y-z, J z-J x\rangle \geq 0, \quad \forall y \in C\right\}
$$

for all $x \in X$. Then

(i) $T_{r}$ is single-valued;

(ii) $T_{r}$ is a firmly nonexpansive-type mapping, that is, for all $x, y \in X$,

$$
\left\langle T_{r} x-T_{r} y, J T_{r} x-J T_{r} y\right\rangle \leq\left\langle T_{r} x-T_{r} y, J x-J y\right\rangle
$$

(iii) $F\left(T_{r}\right)=\widehat{F}\left(T_{r}\right)=E P(f)$;

(iv) $E P(f)$ is closed and convex.

Using Lemma 2.9, we have the following result.

Lemma 2.10 (see [18]). Let $C$ be a nonempty closed convex subset of a smooth, strictly convex and reflexive Banach space $X, f: C \times C \rightarrow \mathbb{R}$ a bifunction satisfying conditions (A1)-(A4), and $r>0$. Then, for $x \in X$ and $q \in F\left(T_{r}\right)$,

$$
\phi\left(q, T_{r} x\right)+\phi\left(T_{r} x, x\right) \leq \phi(q, x) .
$$

Utilizing Lemmas 2.8, 2.9, and 2.10, Zhang [1] derived the following result.

Proposition 2.11 (see [1]). Let X be a smooth, strictly convex and reflexive Banach space and let $C$ be a nonempty closed convex subset of $X$. Let $A: C \rightarrow X^{*}$ be an $\alpha$-inverse-strongly monotone mapping, $f: C \times C \rightarrow \mathbb{R}$ a bifunction satisfying conditions (A1)-(A4), and $r>0$. Then

(I) for $x \in X$, there exists $u \in C$ such that

$$
f(u, y)+\langle A u, y-u\rangle+\frac{1}{r}\langle y-u, J u-J x\rangle \geq 0, \quad \forall y \in C
$$

(II) if $X$ is additionally uniformly smooth and $K_{r}: C \rightarrow C$ is defined as

$$
K_{r}(x)=\left\{u \in C: f(u, y)+\langle A u, y-u\rangle+\frac{1}{r}\langle y-u, J u-J x\rangle \geq 0, \forall y \in C\right\}, \quad \forall x \in C,
$$

then the mapping $K_{r}$ has the following properties:

(i) $K_{r}$ is single-valued,

(ii) $K_{r}$ is a firmly nonexpansive-type mapping, that is,

$$
\left\langle K_{r} x-K_{r} y, J K_{r} x-J K_{r} y\right\rangle \leq\left\langle K_{r} x-K_{r} y, J x-J y\right\rangle, \quad \forall x, y \in X,
$$


Fixed Point Theory and Applications

(iii) $F\left(K_{r}\right)=\widehat{F}\left(K_{r}\right)=E P$,

(iv) EP is a closed convex subset of $C$,

(v) $\phi\left(p, K_{r} x\right)+\phi\left(K_{r} x, x\right) \leq \phi(p, x)$, for all $p \in F\left(K_{r}\right)$.

Proof. Define a bifunction $F: C \times C \rightarrow \mathbb{R}$ by

$$
F(x, y)=f(x, y)+\langle A x, y-x\rangle, \quad \forall x, y \in C .
$$

It is easy to verify that $F$ satisfies the conditions (A1)-(A4). Therefore, the conclusions (I) and (II) follow immediately from Lemmas 2.8, 2.9, and 2.10.

Let $T, \tilde{T}: X \rightarrow 2^{X^{*}}$ be two maximal monotone operators in a smooth Banach space $X$. We denote the resolvent operators of $T$ and $\tilde{T}$ by $J_{r}=(J+r T)^{-1} J$ and $\tilde{J}_{r}=(J+r \tilde{T})^{-1} J$ for each $r>0$, respectively. Then $J_{r}: X \rightarrow D(T)$ and $\tilde{J}_{r}: X \rightarrow D(\widetilde{T})$ are two single-valued mappings. Also, $T^{-1} 0=F\left(J_{r}\right)$ and $\widetilde{T}^{-1} 0=F\left(\tilde{J}_{r}\right)$ for each $r>0$, where $F\left(J_{r}\right)$ and $F\left(\widetilde{J}_{r}\right)$ are the sets of fixed points of $J_{r}$ and $\widetilde{J}_{r}$, respectively. For each $r>0$, the Yosida approximations of $T$ and $\widetilde{T}$ are defined by $A_{r}=\left(J-J J_{r}\right) / r$ and $\tilde{A}_{r}=\left(J-J \tilde{J}_{r}\right) / r$, respectively. It is known that

$$
A_{r} x \in T\left(J_{r} x\right), \quad \tilde{A}_{r} x \in \tilde{T}\left(\tilde{J}_{r} x\right), \quad \text { for each } r>0, x \in X
$$

Lemma 2.12 (see [23]). Let $X$ be a reflexive, strictly convex and smooth Banach space, and let $T$ : $X \rightarrow 2^{X^{*}}$ be a maximal monotone operator with $T^{-1} 0 \neq \emptyset$. Then,

$$
\phi\left(z, J_{r} x\right)+\phi\left(J_{r} x, x\right) \leq \phi(z, x), \quad \forall r>0, z \in T^{-1} 0, x \in X .
$$

Lemma 2.13 (see [36]). Let $\left\{a_{n}\right\}$ and $\left\{b_{n}\right\}$ be two sequences of nonnegative real numbers such that $a_{n+1} \leq a_{n}+b_{n}$ for all $n \geq 0$. If $\sum_{n=0}^{\infty} b_{n}<\infty$, then $\lim _{n \rightarrow \infty} a_{n}$ exists.

\section{Strong Convergence Theorem}

In this section, we prove a strong convergence theorem for finding a common element of the set of solutions for a generalized equilibrium problem and the set $T^{-1} 0 \cap \widetilde{T}^{-1} 0$ for two maximal monotone operators $T$ and $\widetilde{T}$.

Theorem 3.1. Suppose that Assumption 2.1 is satisfied. Let $x_{0} \in X$ be chosen arbitrarily. Consider the sequence

$$
x_{n+1}=\Pi_{H_{n} \cap W_{n}} x_{0}, \quad n=0,1,2, \ldots,
$$


where

$$
\begin{gathered}
H_{n}=\left\{z \in C: \phi\left(z, K_{r_{n}} y_{n}\right) \leq\left(\alpha_{n}+\widetilde{\alpha}_{n}-\alpha_{n} \tilde{\alpha}_{n}\right) \phi\left(z, x_{0}\right)+\left(1-\alpha_{n}\right)\left(1-\widetilde{\alpha}_{n}\right) \phi\left(z, x_{n}\right)\right\}, \\
W_{n}=\left\{z \in C:\left\langle x_{n}-z, J x_{0}-J x_{n}\right\rangle \geq 0\right\}, \\
\tilde{x}_{n}=J^{-1}\left(\alpha_{n} J x_{0}+\left(1-\alpha_{n}\right)\left(\beta_{n} J x_{n}+\left(1-\beta_{n}\right) J J_{r_{n}} x_{n}\right)\right), \\
y_{n}=J^{-1}\left(\widetilde{\alpha}_{n} J x_{0}+\left(1-\widetilde{\alpha}_{n}\right)\left(\widetilde{\beta}_{n} J \widetilde{x}_{n}+\left(1-\widetilde{\beta}_{n}\right) J \widetilde{J}_{r_{n}} \widetilde{x}_{n}\right)\right),
\end{gathered}
$$

$K_{r}$ is defined by (2.14), $\left\{\alpha_{n}\right\},\left\{\beta_{n}\right\},\left\{\tilde{\alpha}_{n}\right\},\left\{\tilde{\beta}_{n}\right\} \subset[0,1]$ satisfy

$$
\lim _{n \rightarrow \infty} \alpha_{n}=0, \quad \lim _{n \rightarrow \infty} \tilde{\alpha}_{n}=0, \quad \liminf _{n \rightarrow \infty} \beta_{n}\left(1-\beta_{n}\right)>0, \quad \liminf _{n \rightarrow \infty} \tilde{\beta}_{n}\left(1-\tilde{\beta}_{n}\right)>0,
$$

and $\left\{r_{n}\right\} \subset(0, \infty)$ satisfies $\liminf _{n \rightarrow \infty} r_{n}>0$. Then, the sequence $\left\{x_{n}\right\}$ converges strongly to $\Pi_{T^{-1} 0 \cap \tilde{T}^{-1} 0 \cap E P} x_{0}$, where $\Pi_{T^{-1} 0 \cap \tilde{T}^{-1} O \cap E P}$ is the generalized projection of $X$ onto $T^{-1} 0 \cap \widetilde{T}^{-1} 0 \cap E P$.

Proof. For the sake of simplicity, we define

$$
u_{n}:=K_{r_{n}} y_{n}, \quad z_{n}:=J^{-1}\left(\beta_{n} J x_{n}+\left(1-\beta_{n}\right) J J_{r_{n}} x_{n}\right), \quad \tilde{z}_{n}:=J^{-1}\left(\tilde{\beta}_{n} J \tilde{x}_{n}+\left(1-\tilde{\beta}_{n}\right) J \tilde{J}_{r_{n}} \tilde{x}_{n}\right),
$$

so that

$$
\tilde{x}_{n}=J^{-1}\left(\alpha_{n} J x_{0}+\left(1-\alpha_{n}\right) J z_{n}\right), \quad y_{n}=J^{-1}\left(\widetilde{\alpha}_{n} J x_{0}+\left(1-\widetilde{\alpha}_{n}\right) J \widetilde{z}_{n}\right) .
$$

We divide the proof into several steps.

Step 1. We claim that $H_{n} \cap W_{n}$ is closed and convex for each $n \geq 0$.

Indeed, it is obvious that $H_{n}$ is closed and $W_{n}$ is closed and convex for each $n \geq 0$. Let us show that $H_{n}$ is convex. For $z_{1}, z_{2} \in H_{n}$ and $t \in(0,1)$, put $z=t z_{1}+(1-t) z_{2}$. It is sufficient to show that $z \in H_{n}$. We first write $\gamma_{n}=\alpha_{n}+\tilde{\alpha}_{n}-\alpha_{n} \tilde{\alpha}_{n}$ for each $n \geq 0$. Next, we prove that

$$
\phi\left(z, u_{n}\right) \leq \gamma_{n} \phi\left(z, x_{0}\right)+\left(1-\gamma_{n}\right) \phi\left(z, x_{n}\right)
$$

is equivalent to

$$
2 \gamma_{n}\left\langle z, J x_{0}\right\rangle+2\left(1-\gamma_{n}\right)\left\langle z, J x_{n}\right\rangle-2\left\langle z, J u_{n}\right\rangle \leq \gamma_{n}\left\|x_{0}\right\|^{2}+\left(1-\gamma_{n}\right)\left\|x_{n}\right\|^{2}-\left\|u_{n}\right\|^{2} .
$$

Indeed, from (2.1) we deduce that there hold the following:

$$
\begin{aligned}
& \phi\left(z, x_{0}\right)=\|z\|^{2}-2\left\langle z, J x_{0}\right\rangle+\left\|x_{0}\right\|^{2}, \\
& \phi\left(z, x_{n}\right)=\|z\|^{2}-2\left\langle z, J x_{n}\right\rangle+\left\|x_{n}\right\|^{2}, \\
& \phi\left(z, u_{n}\right)=\|z\|^{2}-2\left\langle z, J u_{n}\right\rangle+\left\|u_{n}\right\|^{2},
\end{aligned}
$$


which combined with (3.6) yield that (3.6) is equivalent to (3.7). Thus we have

$$
\begin{aligned}
2 \gamma_{n}\left\langle z, J x_{0}\right\rangle+2\left(1-\gamma_{n}\right)\left\langle z, J x_{n}\right\rangle-2\left\langle z, J u_{n}\right\rangle \\
=2 \gamma_{n}\left\langle t z_{1}+(1-t) z_{2}, J x_{0}\right\rangle+2\left(1-\gamma_{n}\right)\left\langle t z_{1}+(1-t) z_{2}, J x_{n}\right\rangle \\
\quad-2\left\langle t z_{1}+(1-t) z_{2}, J u_{n}\right\rangle \\
=2 t \gamma_{n}\left\langle z_{1}, J x_{0}\right\rangle+2(1-t) \gamma_{n}\left\langle z_{2}, J x_{0}\right\rangle+2\left(1-\gamma_{n}\right) t\left\langle z_{1}, J x_{n}\right\rangle \\
\quad+2\left(1-\gamma_{n}\right)(1-t)\left\langle z_{2}, J x_{n}\right\rangle-2 t\left\langle z_{1}, J u_{n}\right\rangle-2(1-t)\left\langle z_{2}, J u_{n}\right\rangle \\
\leq \gamma_{n}\left\|x_{0}\right\|^{2}+\left(1-\gamma_{n}\right)\left\|x_{n}\right\|^{2}-\left\|u_{n}\right\|^{2} .
\end{aligned}
$$

This implies that $z \in H_{n}$. Therefore, $H_{n}$ is closed and convex.

Step 2. We claim that $T^{-1} 0 \cap \widetilde{T}^{-1} 0 \cap E P \subset H_{n} \cap W_{n}$ for each $n \geq 0$ and that $\left\{x_{n}\right\}$ is well defined. Indeed, take $w \in T^{-1} 0 \cap \tilde{T}^{-1} 0 \cap E P$ arbitrarily. Note that $u_{n}=K_{r_{n}} y_{n}$ is equivalent to

$$
u_{n} \in C \text { such that } f\left(u_{n}, y\right)+\left\langle A u_{n}, y-u_{n}\right\rangle+\frac{1}{r_{n}}\left\langle y-u_{n}, J u_{n}-J y_{n}\right\rangle \geq 0, \quad \forall y \in C \text {. }
$$

Then from Lemma 2.12, we obtain

$$
\begin{aligned}
\phi\left(w, z_{n}\right) & =\phi\left(w, J^{-1}\left(\beta_{n} J x_{n}+\left(1-\beta_{n}\right) J J_{r_{n}} x_{n}\right)\right) \\
& =\|w\|^{2}-2\left\langle w, \beta_{n} J x_{n}+\left(1-\beta_{n}\right) J J_{r_{n}} x_{n}\right\rangle+\left\|\beta_{n} J x_{n}+\left(1-\beta_{n}\right) J J_{r_{n}} x_{n}\right\|^{2} \\
& \leq\|w\|^{2}-2 \beta_{n}\left\langle w, J x_{n}\right\rangle-2\left(1-\beta_{n}\right)\left\langle w, J J_{r_{n}} x_{n}\right\rangle+\beta_{n}\left\|x_{n}\right\|^{2}+\left(1-\beta_{n}\right)\left\|J_{r_{n}} x_{n}\right\|^{2} \\
& =\beta_{n} \phi\left(w, x_{n}\right)+\left(1-\beta_{n}\right) \phi\left(w, J_{r_{n}} x_{n}\right) \\
& \leq \beta_{n} \phi\left(w, x_{n}\right)+\left(1-\beta_{n}\right) \phi\left(w, x_{n}\right)=\phi\left(w, x_{n}\right) \\
\phi\left(w, \tilde{x}_{n}\right) & =\phi\left(w, J^{-1}\left(\alpha_{n} J x_{0}+\left(1-\alpha_{n}\right) J z_{n}\right)\right) \\
& =\|w\|^{2}-2\left\langle w, \alpha_{n} J x_{0}+\left(1-\alpha_{n}\right) J z_{n}\right\rangle+\left\|\alpha_{n} J x_{0}+\left(1-\alpha_{n}\right) J z_{n}\right\|^{2} \\
& \leq\|w\|^{2}-2 \alpha_{n}\left\langle w, J x_{0}\right\rangle-2\left(1-\alpha_{n}\right)\left\langle w, J z_{n}\right\rangle+\alpha_{n}\left\|x_{0}\right\|^{2}+\left(1-\alpha_{n}\right)\left\|z_{n}\right\|^{2} \\
& =\alpha_{n} \phi\left(w, x_{0}\right)+\left(1-\alpha_{n}\right) \phi\left(w, z_{n}\right) \\
& \leq \alpha_{n} \phi\left(w, x_{0}\right)+\left(1-\alpha_{n}\right) \phi\left(w, x_{n}\right) .
\end{aligned}
$$


Moreover, we have

$$
\begin{aligned}
& \phi\left(w, \tilde{z}_{n}\right)=\phi\left(w, J^{-1}\left(\tilde{\beta}_{n} J \tilde{x}_{n}+\left(1-\tilde{\beta}_{n}\right) J \tilde{J}_{r_{n}} \tilde{x}_{n}\right)\right) \\
& \leq \tilde{\beta}_{n} \phi\left(w, \tilde{x}_{n}\right)+\left(1-\tilde{\beta}_{n}\right) \phi\left(w, \tilde{J}_{r_{n}} \tilde{x}_{n}\right) \\
& \leq \tilde{\beta}_{n} \phi\left(w, \tilde{x}_{n}\right)+\left(1-\tilde{\beta}_{n}\right) \phi\left(w, \tilde{x}_{n}\right)=\phi\left(w, \tilde{x}_{n}\right), \\
& \phi\left(w, y_{n}\right)=\phi\left(w, J^{-1}\left(\tilde{\alpha}_{n} J x_{0}+\left(1-\tilde{\alpha}_{n}\right) J \widetilde{z}_{n}\right)\right) \\
& \leq\|w\|^{2}-2 \tilde{\alpha}_{n}\left\langle w, J x_{0}\right\rangle-2\left(1-\tilde{\alpha}_{n}\right)\left\langle w, J \tilde{z}_{n}\right\rangle+\tilde{\alpha}_{n}\left\|x_{0}\right\|^{2}+\left(1-\tilde{\alpha}_{n}\right)\left\|\tilde{z}_{n}\right\|^{2} \\
& =\tilde{\alpha}_{n} \phi\left(w, x_{0}\right)+\left(1-\tilde{\alpha}_{n}\right) \phi\left(w, \widetilde{z}_{n}\right) \\
& \leq \tilde{\alpha}_{n} \phi\left(w, x_{0}\right)+\left(1-\tilde{\alpha}_{n}\right) \phi\left(w, \tilde{x}_{n}\right) \\
& \leq \tilde{\alpha}_{n} \phi\left(w, x_{0}\right)+\left(1-\tilde{\alpha}_{n}\right)\left[\alpha_{n} \phi\left(w, x_{0}\right)+\left(1-\alpha_{n}\right) \phi\left(w, x_{n}\right)\right] \\
& =\left[\tilde{\alpha}_{n}+\left(1-\tilde{\alpha}_{n}\right) \alpha_{n}\right] \phi\left(w, x_{0}\right)+\left(1-\tilde{\alpha}_{n}\right)\left(1-\alpha_{n}\right) \phi\left(w, x_{n}\right) \\
& \leq\left(\alpha_{n}+\tilde{\alpha}_{n}-\alpha_{n} \tilde{\alpha}_{n}\right) \phi\left(w, x_{0}\right)+\left(1-\alpha_{n}\right)\left(1-\tilde{\alpha}_{n}\right) \phi\left(w, x_{n}\right),
\end{aligned}
$$

and hence by Proposition 2.11,

$$
\begin{aligned}
\phi\left(w, u_{n}\right) & =\phi\left(w, K_{r_{n}} y_{n}\right) \leq \phi\left(w, y_{n}\right) \\
& \leq\left(\alpha_{n}+\widetilde{\alpha}_{n}-\alpha_{n} \widetilde{\alpha}_{n}\right) \phi\left(w, x_{0}\right)+\left(1-\alpha_{n}\right)\left(1-\widetilde{\alpha}_{n}\right) \phi\left(w, x_{n}\right) .
\end{aligned}
$$

So $w \in H_{n}$ for all $n \geq 0$. Now, let us show that

$$
T^{-1} 0 \cap \widetilde{T}^{-1} 0 \cap E P \subset W_{n} \quad \forall n \geq 0 .
$$

We prove this by induction. For $n=0$, we have $T^{-1} 0 \cap \tilde{T}^{-1} 0 \cap E P \subset C=W_{0}$. Assume that $T^{-1} 0 \cap \widetilde{T}^{-1} 0 \cap E P \subset W_{n}$. Since $x_{n+1}$ is the projection of $x_{0}$ onto $H_{n} \cap W_{n}$, by Lemma 2.3 we have

$$
\left\langle x_{n+1}-z, J x_{0}-J x_{n+1}\right\rangle \geq 0, \quad \forall z \in H_{n} \cap W_{n} .
$$

As $T^{-1} 0 \cap \widetilde{T}^{-1} 0 \cap E P \subset H_{n} \cap W_{n}$ by the induction assumption, the last inequality holds, in particular, for all $z \in T^{-1} 0 \cap \widetilde{T}^{-1} 0 \cap E P$. This, together with the definition of $W_{n+1}$ implies that $T^{-1} 0 \cap \widetilde{T}^{-1} 0 \cap E P \subset W_{n+1}$. Hence (3.14) holds for all $n \geq 0$. So, $T^{-1} 0 \cap \widetilde{T}^{-1} 0 \cap E P \subset H_{n} \cap W_{n}$ for all $n \geq 0$. This implies that the sequence $\left\{x_{n}\right\}$ is well defined.

Step 3. We claim that $\left\{x_{n}\right\}$ is bounded and that $\phi\left(x_{n+1}, x_{n}\right) \rightarrow 0$ as $n \rightarrow \infty$.

Indeed, it follows from the definition of $W_{n}$ that $x_{n}=\Pi_{W_{n}} x_{0}$. Since $x_{n}=\Pi_{W_{n}} x_{0}$ and $x_{n+1}=\Pi_{H_{n} \cap W_{n}} x_{0} \in W_{n}$, so $\phi\left(x_{n}, x_{0}\right) \leq \phi\left(x_{n+1}, x_{0}\right)$ for all $n \geq 0$, that is, $\left\{\phi\left(x_{n}, x_{0}\right)\right\}$ is nondecreasing. It follows from $x_{n}=\Pi_{W_{n}} x_{0}$ and Lemma 2.4 that

$$
\phi\left(x_{n}, x_{0}\right)=\phi\left(\Pi_{W_{n}} x_{0}, x_{0}\right) \leq \phi\left(p, x_{0}\right)-\phi\left(p, x_{n}\right) \leq \phi\left(p, x_{0}\right)
$$


for each $p \in T^{-1} 0 \cap \tilde{T}^{-1} 0 \cap E P \subset W_{n}$ for each $n \geq 0$. Therefore, $\left\{\phi\left(x_{n}, x_{0}\right)\right\}$ is bounded, which implies that the limit of $\left\{\phi\left(x_{n}, x_{0}\right)\right\}$ exists. Since

$$
\left(\left\|x_{n}\right\|-\left\|x_{0}\right\|\right)^{2} \leq \phi\left(x_{n}, x_{0}\right) \leq\left(\left\|x_{n}\right\|+\left\|x_{0}\right\|\right)^{2}, \quad \forall n \geq 0,
$$

so $\left\{x_{n}\right\}$ is bounded. From Lemma 2.4, we have

$$
\begin{aligned}
\phi\left(x_{n+1}, x_{n}\right) & =\phi\left(x_{n+1}, \Pi_{W_{n}} x_{0}\right) \leq \phi\left(x_{n+1}, x_{0}\right)-\phi\left(\Pi_{W_{n}} x_{0}, x_{0}\right) \\
& =\phi\left(x_{n+1}, x_{0}\right)-\phi\left(x_{n}, x_{0}\right)
\end{aligned}
$$

for each $n \geq 0$. This implies that

$$
\lim _{n \rightarrow \infty} \phi\left(x_{n+1}, x_{n}\right)=0
$$

Step 4. We claim that $\lim _{n \rightarrow \infty}\left\|x_{n}-u_{n}\right\|=0, \lim _{n \rightarrow \infty}\left\|x_{n}-J_{r_{n}} x_{n}\right\|=0$, and $\lim _{n \rightarrow \infty}\left\|\tilde{x}_{n}-\widetilde{J}_{r_{n}} \tilde{x}_{n}\right\|=$ 0 .

Indeed, from $x_{n+1}=\Pi_{H_{n} \cap W_{n}} x_{0} \in H_{n}$, we have

$$
\phi\left(x_{n+1}, u_{n}\right) \leq\left(\alpha_{n}+\tilde{\alpha}_{n}-\alpha_{n} \tilde{\alpha}_{n}\right) \phi\left(x_{n+1}, x_{0}\right)+\left(1-\alpha_{n}\right)\left(1-\tilde{\alpha}_{n}\right) \phi\left(x_{n+1}, x_{n}\right), \quad \forall n \geq 0 .
$$

Therefore, from $\alpha_{n} \rightarrow 0 \widetilde{\alpha}_{n} \rightarrow 0$ and $\phi\left(x_{n+1}, x_{n}\right) \rightarrow 0$, it follows that $\lim _{n \rightarrow \infty} \phi\left(x_{n+1}, u_{n}\right)=0$.

Since $\lim _{n \rightarrow \infty} \phi\left(x_{n+1}, x_{n}\right)=\lim _{n \rightarrow \infty} \phi\left(x_{n+1}, u_{n}\right)=0$ and $X$ is uniformly convex and smooth, we have from Lemma 2.2 that

$$
\lim _{n \rightarrow \infty}\left\|x_{n+1}-x_{n}\right\|=\lim _{n \rightarrow \infty}\left\|x_{n+1}-u_{n}\right\|=0,
$$

and, therefore, $\lim _{n \rightarrow \infty}\left\|x_{n}-u_{n}\right\|=0$. Since $J$ is uniformly norm-to-norm continuous on bounded subsets of $X$ and $\left\|x_{n}-u_{n}\right\| \rightarrow 0$, then $\lim _{n \rightarrow \infty}\left\|J x_{n}-J u_{n}\right\|=0$.

Let us set $\Omega:=T^{-1} 0 \cap \widetilde{T}^{-1} 0 \cap E P$. Then, according to Lemma 2.5 and Proposition 2.11, we know that $\Omega$ is a nonempty closed convex subset of $X$ such that $\Omega \subset C$. Fix $u \in \Omega$ arbitrarily. As in the proof of Step 2, we can show that $\phi\left(u, z_{n}\right) \leq \phi\left(u, x_{n}\right)$,

$$
\begin{gathered}
\phi\left(u, \tilde{x}_{n}\right) \leq \alpha_{n} \phi\left(u, x_{0}\right)+\left(1-\alpha_{n}\right) \phi\left(u, x_{n}\right), \\
\phi\left(u, \tilde{z}_{n}\right) \leq \phi\left(u, \tilde{x}_{n}\right), \\
\phi\left(u, y_{n}\right) \leq\left(\alpha_{n}+\tilde{\alpha}_{n}-\alpha_{n} \widetilde{\alpha}_{n}\right) \phi\left(u, x_{0}\right)+\left(1-\alpha_{n}\right)\left(1-\tilde{\alpha}_{n}\right) \phi\left(u, x_{n}\right), \\
\phi\left(u, u_{n}\right) \leq\left(\alpha_{n}+\tilde{\alpha}_{n}-\alpha_{n} \tilde{\alpha}_{n}\right) \phi\left(u, x_{0}\right)+\left(1-\alpha_{n}\right)\left(1-\tilde{\alpha}_{n}\right) \phi\left(u, x_{n}\right) .
\end{gathered}
$$


Hence it follows from the boundedness of $\left\{x_{n}\right\}$ that $\left\{z_{n}\right\},\left\{\tilde{x}_{n}\right\},\left\{\tilde{z}_{n}\right\},\left\{y_{n}\right\}$, and $\left\{u_{n}\right\}$ are also bounded. Let $r=\sup \left\{\left\|x_{n}\right\|,\left\|\tilde{x}_{n}\right\|,\left\|J_{r_{n}} x_{n}\right\|,\left\|\widetilde{J}_{r_{n}} \widetilde{x}_{n}\right\|: n \geq 0\right\}$. Since $X$ is a uniformly smooth Banach space, we know that $X^{*}$ is a uniformly convex Banach space. Therefore, by Lemma 2.6 there exists a continuous, strictly increasing, and convex function $g$, with $g(0)=0$, such that

$$
\left\|\alpha x^{*}+(1-\alpha) y^{*}\right\|^{2} \leq \alpha\left\|x^{*}\right\|^{2}+(1-\alpha)\left\|y^{*}\right\|^{2}-\alpha(1-\alpha) g\left(\left\|x^{*}-y^{*}\right\|\right),
$$

for $x^{*}, y^{*} \in B_{r}^{*}$ and $\alpha \in[0,1]$. So, we have that

$$
\begin{aligned}
& \phi\left(u, z_{n}\right)=\phi\left(u, J^{-1}\left(\beta_{n} J x_{n}+\left(1-\beta_{n}\right) J J_{r_{n}} x_{n}\right)\right) \\
& =\|u\|^{2}-2\left\langle u, \beta_{n} J x_{n}+\left(1-\beta_{n}\right) J J_{r_{n}} x_{n}\right\rangle+\left\|\beta_{n} J x_{n}+\left(1-\beta_{n}\right) J J_{r_{n}} x_{n}\right\|^{2} \\
& \leq\|u\|^{2}-2 \beta_{n}\left\langle u, J x_{n}\right\rangle-2\left(1-\beta_{n}\right)\left\langle u, J J_{r_{n}} x_{n}\right\rangle \\
& +\beta_{n}\left\|x_{n}\right\|^{2}+\left(1-\beta_{n}\right)\left\|J_{r_{n}} x_{n}\right\|^{2}-\beta_{n}\left(1-\beta_{n}\right) g\left(\left\|J x_{n}-J J_{r_{n}} x_{n}\right\|\right) \\
& =\beta_{n} \phi\left(u, x_{n}\right)+\left(1-\beta_{n}\right) \phi\left(u, J_{r_{n}} x_{n}\right)-\beta_{n}\left(1-\beta_{n}\right) g\left(\left\|J x_{n}-J J_{r_{n}} x_{n}\right\|\right) \\
& \leq \beta_{n} \phi\left(u, x_{n}\right)+\left(1-\beta_{n}\right) \phi\left(u, x_{n}\right)-\beta_{n}\left(1-\beta_{n}\right) g\left(\left\|J x_{n}-J J_{r_{n}} x_{n}\right\|\right) \\
& =\phi\left(u, x_{n}\right)-\beta_{n}\left(1-\beta_{n}\right) g\left(\left\|J x_{n}-J J_{r_{n}} x_{n}\right\|\right), \\
& \phi\left(u, \tilde{z}_{n}\right)=\phi\left(u, J^{-1}\left(\tilde{\beta}_{n} J \tilde{x}_{n}+\left(1-\tilde{\beta}_{n}\right) J \tilde{J}_{r_{n}} \tilde{x}_{n}\right)\right) \\
& =\|u\|^{2}-2\left\langle u, \tilde{\beta}_{n} J \tilde{x}_{n}+\left(1-\tilde{\beta}_{n}\right) J \tilde{J}_{r_{n}} \tilde{x}_{n}\right\rangle+\left\|\tilde{\beta}_{n} J \tilde{x}_{n}+\left(1-\tilde{\beta}_{n}\right) J \tilde{J}_{r_{n}} \tilde{x}_{n}\right\|^{2} \\
& \leq\|u\|^{2}-2 \tilde{\beta}_{n}\left\langle u, J \tilde{x}_{n}\right\rangle-2\left(1-\tilde{\beta}_{n}\right)\left\langle u, J \tilde{J}_{r_{n}} \tilde{x}_{n}\right\rangle \\
& +\tilde{\beta}_{n}\left\|\tilde{x}_{n}\right\|^{2}+\left(1-\tilde{\beta}_{n}\right)\left\|\tilde{J}_{r_{n}} \tilde{x}_{n}\right\|^{2}-\tilde{\beta}_{n}\left(1-\tilde{\beta}_{n}\right) g\left(\left\|J \tilde{x}_{n}-J \tilde{J}_{r_{n}} \tilde{x}_{n}\right\|\right) \\
& =\tilde{\beta}_{n} \phi\left(u, \tilde{x}_{n}\right)+\left(1-\tilde{\beta}_{n}\right) \phi\left(u, \widetilde{J}_{r_{n}} \tilde{x}_{n}\right)-\tilde{\beta}_{n}\left(1-\tilde{\beta}_{n}\right) g\left(\left\|J \tilde{x}_{n}-J \tilde{J}_{r_{n}} \tilde{x}_{n}\right\|\right) \\
& \leq \tilde{\beta}_{n} \phi\left(u, \tilde{x}_{n}\right)+\left(1-\tilde{\beta}_{n}\right) \phi\left(u, \tilde{x}_{n}\right)-\tilde{\beta}_{n}\left(1-\tilde{\beta}_{n}\right) g\left(\left\|J \tilde{x}_{n}-J \tilde{J}_{n} \tilde{x}_{n}\right\|\right) \\
& =\phi\left(u, \tilde{x}_{n}\right)-\tilde{\beta}_{n}\left(1-\tilde{\beta}_{n}\right) g\left(\left\|J \tilde{x}_{n}-J \widetilde{J}_{r_{n}} \tilde{x}_{n}\right\|\right),
\end{aligned}
$$


and hence

$$
\begin{aligned}
& \phi\left(u, \tilde{x}_{n}\right)=\phi\left(u, J^{-1}\left(\alpha_{n} J x_{0}+\left(1-\alpha_{n}\right) J z_{n}\right)\right) \\
& =\|u\|^{2}-2\left\langle u, \alpha_{n} J x_{0}+\left(1-\alpha_{n}\right) J z_{n}\right\rangle+\left\|\alpha_{n} J x_{0}+\left(1-\alpha_{n}\right) J z_{n}\right\|^{2} \\
& \leq\|u\|^{2}-2 \alpha_{n}\left\langle u, J x_{0}\right\rangle-2\left(1-\alpha_{n}\right)\left\langle u, J z_{n}\right\rangle+\alpha_{n}\left\|x_{0}\right\|^{2}+\left(1-\alpha_{n}\right)\left\|z_{n}\right\|^{2} \\
& =\alpha_{n} \phi\left(u, x_{0}\right)+\left(1-\alpha_{n}\right) \phi\left(u, z_{n}\right) \\
& \leq \alpha_{n} \phi\left(u, x_{0}\right)+\left(1-\alpha_{n}\right)\left[\phi\left(u, x_{n}\right)-\beta_{n}\left(1-\beta_{n}\right) g\left(\left\|J x_{n}-J J_{r_{n}} x_{n}\right\|\right)\right] \\
& =\alpha_{n} \phi\left(u, x_{0}\right)+\left(1-\alpha_{n}\right) \phi\left(u, x_{n}\right)-\left(1-\alpha_{n}\right) \beta_{n}\left(1-\beta_{n}\right) g\left(\left\|J x_{n}-J J_{r_{n}} x_{n}\right\|\right) \text {, } \\
& \phi\left(u, u_{n}\right)=\phi\left(u, K_{r_{n}} y_{n}\right) \leq \phi\left(u, y_{n}\right) \quad \text { (using Proposition 2.10) } \\
& =\phi\left(u, J^{-1}\left(\tilde{\alpha}_{n} J x_{0}+\left(1-\tilde{\alpha}_{n}\right) J \tilde{z}_{n}\right)\right) \\
& =\|u\|^{2}-2\left\langle u, \widetilde{\alpha}_{n} J x_{0}+\left(1-\widetilde{\alpha}_{n}\right) J \widetilde{z}_{n}\right\rangle+\left\|\widetilde{\alpha}_{n} J x_{0}+\left(1-\widetilde{\alpha}_{n}\right) J \widetilde{z}_{n}\right\|^{2} \\
& \leq\|u\|^{2}-2 \widetilde{\alpha}_{n}\left\langle u, J x_{0}\right\rangle-2\left(1-\widetilde{\alpha}_{n}\right)\left\langle u, J \widetilde{z}_{n}\right\rangle+\tilde{\alpha}_{n}\left\|x_{0}\right\|^{2}+\left(1-\widetilde{\alpha}_{n}\right)\left\|\tilde{z}_{n}\right\|^{2} \\
& =\tilde{\alpha}_{n} \phi\left(u, x_{0}\right)+\left(1-\tilde{\alpha}_{n}\right) \phi\left(u, \tilde{z}_{n}\right) \\
& \leq \tilde{\alpha}_{n} \phi\left(u, x_{0}\right)+\left(1-\tilde{\alpha}_{n}\right)\left[\phi\left(u, \tilde{x}_{n}\right)-\tilde{\beta}_{n}\left(1-\tilde{\beta}_{n}\right) g\left(\left\|J \tilde{x}_{n}-J \tilde{J}_{r_{n}} \tilde{x}_{n}\right\|\right)\right] \\
& =\tilde{\alpha}_{n} \phi\left(u, x_{0}\right)+\left(1-\tilde{\alpha}_{n}\right) \phi\left(u, \tilde{x}_{n}\right)-\left(1-\tilde{\alpha}_{n}\right) \tilde{\beta}_{n}\left(1-\tilde{\beta}_{n}\right) g\left(\left\|J \tilde{x}_{n}-J \tilde{J}_{r_{n}} \tilde{x}_{n}\right\|\right) \\
& \leq \tilde{\alpha}_{n} \phi\left(u, x_{0}\right)+\phi\left(u, \tilde{x}_{n}\right),
\end{aligned}
$$

for all $n \geq 0$. Consequently, we have

$$
\begin{aligned}
(1- & \left.\alpha_{n}\right) \beta_{n}\left(1-\beta_{n}\right) g\left(\left\|J x_{n}-J J_{r_{n}} x_{n}\right\|\right) \\
& \leq \alpha_{n} \varphi\left(u, x_{0}\right)+\left(1-\alpha_{n}\right) \varphi\left(u, x_{n}\right)-\varphi\left(u, \tilde{x}_{n}\right) \\
& \leq \alpha_{n} \varphi\left(u, x_{0}\right)+\varphi\left(u, x_{n}\right)-\varphi\left(u, \tilde{x}_{n}\right) \\
& =\alpha_{n} \varphi\left(u, x_{0}\right)+\varphi\left(u, x_{n}\right)-\varphi\left(u, u_{n}\right)+\varphi\left(u, u_{n}\right)-\varphi\left(u, \tilde{x}_{n}\right) \\
& =\alpha_{n} \varphi\left(u, x_{0}\right)+\left\|x_{n}\right\|^{2}-\left\|u_{n}\right\|^{2}-2\left\langle u, J x_{n}-J u_{n}\right\rangle+\varphi\left(u, u_{n}\right)-\varphi\left(u, \tilde{x}_{n}\right) \\
& \leq \alpha_{n} \varphi\left(u, x_{0}\right)+\left|\left\|x_{n}\right\|^{2}-\left\|u_{n}\right\|^{2}\right|+2\left|\left\langle u, J x_{n}-J u_{n}\right\rangle\right|+\varphi\left(u, u_{n}\right)-\varphi\left(u, \tilde{x}_{n}\right) \\
& \leq \alpha_{n} \varphi\left(u, x_{0}\right)+\tilde{\alpha}_{n} \varphi\left(u, x_{0}\right)+\mid\left\|x_{n}\right\|-\left\|u_{n}\right\|\left(\left\|x_{n}\right\|+\left\|u_{n}\right\|\right)+2\|u\|\left\|J x_{n}-J u_{n}\right\| \\
& \leq\left(\alpha_{n}+\tilde{\alpha}_{n}\right) \varphi\left(u, x_{0}\right)+\left\|x_{n}-u_{n}\right\|\left(\left\|x_{n}\right\|+\left\|u_{n}\right\|\right)+2\|u\|\left\|J x_{n}-J u_{n}\right\|,
\end{aligned}
$$


Since $\left\|x_{n}-u_{n}\right\| \rightarrow 0$ and $J$ is uniformly norm-to-norm continuous on bounded subsets of $X$, we obtain $\left\|J x_{n}-J u_{n}\right\| \rightarrow 0$. From $\lim _{\inf _{n \rightarrow \infty}} \beta_{n}\left(1-\beta_{n}\right)>0$ and $\lim _{n \rightarrow \infty}\left(\alpha_{n}+\tilde{\alpha}_{n}\right)=0$, we have

$$
\lim _{n \rightarrow \infty} g\left(\left\|J x_{n}-J J_{r_{n}} x_{n}\right\|\right)=0 .
$$

Therefore, from the properties of $g$, we get

$$
\lim _{n \rightarrow \infty}\left\|J x_{n}-J J_{r_{n}} x_{n}\right\|=\lim _{n \rightarrow \infty}\left\|x_{n}-J_{r_{n}} x_{n}\right\|=0
$$

recalling that $J^{-1}$ is uniformly norm-to-norm continuous on bounded subsets of $X^{*}$. Next let us show that

$$
\lim _{n \rightarrow \infty}\left\|J \tilde{x}_{n}-J \tilde{J}_{r_{n}} \tilde{x}_{n}\right\|=\lim _{n \rightarrow \infty}\left\|\tilde{x}_{n}-\tilde{J}_{r_{n}} \tilde{x}_{n}\right\|=0 .
$$

Observe first that

$$
\begin{aligned}
\phi\left(u_{n},\right. & \left.x_{n}\right)-\phi\left(x_{n+1}, u_{n}\right) \\
& =\left\|x_{n}\right\|^{2}-\left\|x_{n+1}\right\|^{2}-2\left\langle u_{n}, J x_{n}\right\rangle+2\left\langle x_{n+1}, J u_{n}\right\rangle \\
& =\left(\left\|x_{n}\right\|-\left\|x_{n+1}\right\|\right)\left(\left\|x_{n}\right\|+\left\|x_{n+1}\right\|\right)+2\left\langle x_{n+1}-u_{n}, J x_{n}\right\rangle+2\left\langle x_{n+1}, J u_{n}-J x_{n}\right\rangle \\
& \leq\left\|x_{n}-x_{n+1}\right\|\left(\left\|x_{n}\right\|+\left\|x_{n+1}\right\|\right)+2\left\|x_{n+1}-u_{n}\right\|\left\|x_{n}\right\|+2\left\|x_{n+1}\right\|\left\|J u_{n}-J x_{n}\right\| .
\end{aligned}
$$

Since $\phi\left(x_{n+1}, u_{n}\right) \rightarrow 0,\left\|x_{n+1}-x_{n}\right\| \rightarrow 0,\left\|x_{n+1}-u_{n}\right\| \rightarrow 0,\left\|J u_{n}-J x_{n}\right\| \rightarrow 0$, and $\left\{x_{n}\right\}$ is bounded, so it follows that $\phi\left(u_{n}, x_{n}\right) \rightarrow 0$. Also, observe that

$$
\begin{aligned}
\phi\left(u_{n}, J_{r_{n}} x_{n}\right)-\phi\left(u_{n}, x_{n}\right) & =\left\|J_{r_{n}} x_{n}\right\|^{2}-\left\|x_{n}\right\|^{2}+2\left\langle u_{n}, J x_{n}-J J_{r_{n}} x_{n}\right\rangle \\
& =\left(\left\|J_{r_{n}} x_{n}\right\|-\left\|x_{n}\right\|\right)\left(\left\|J_{r_{n}} x_{n}\right\|+\left\|x_{n}\right\|\right)+2\left\langle u_{n}, J x_{n}-J J_{r_{n}} x_{n}\right\rangle \\
& \leq\left\|J_{r_{n}} x_{n}-x_{n}\right\|\left(\left\|J_{r_{n}} x_{n}\right\|+\left\|x_{n}\right\|\right)+2\left\|u_{n}\right\|\left\|J x_{n}-J J_{r_{n}} x_{n}\right\| .
\end{aligned}
$$

Since $\phi\left(u_{n}, x_{n}\right) \rightarrow 0,\left\|J_{r_{n}} x_{n}-x_{n}\right\| \rightarrow 0,\left\|J x_{n}-J J_{r_{n}} x_{n}\right\| \rightarrow 0$, and the sequences $\left\{x_{n}\right\},\left\{u_{n}\right\},\left\{J_{r_{n}} x_{n}\right\}$ are bounded, so it follows that $\phi\left(u_{n}, J_{r_{n}} x_{n}\right) \rightarrow 0$. Meantime, observe that

$$
\begin{aligned}
\phi\left(u_{n}, z_{n}\right) & =\phi\left(u_{n}, J^{-1}\left(\beta_{n} J x_{n}+\left(1-\beta_{n}\right) J J_{r_{n}} x_{n}\right)\right) \\
& =\left\|u_{n}\right\|^{2}-2\left\langle u_{n}, \beta_{n} J x_{n}+\left(1-\beta_{n}\right) J J_{r_{n}} x_{n}\right\rangle+\left\|\beta_{n} J x_{n}+\left(1-\beta_{n}\right) J J_{r_{n}} x_{n}\right\|^{2} \\
& \leq\left\|u_{n}\right\|^{2}-2 \beta_{n}\left\langle u_{n}, J x_{n}\right\rangle-2\left(1-\beta_{n}\right)\left\langle u_{n}, J J_{r_{n}} x_{n}\right\rangle+\beta_{n}\left\|x_{n}\right\|^{2}+\left(1-\beta_{n}\right)\left\|J_{r_{n}} x_{n}\right\|^{2} \\
& =\beta_{n} \phi\left(u_{n}, x_{n}\right)+\left(1-\beta_{n}\right) \phi\left(u_{n}, J_{r_{n}} x_{n}\right) \\
& \leq \phi\left(u_{n}, x_{n}\right)+\phi\left(u_{n}, J_{r_{n}} x_{n}\right)
\end{aligned}
$$


and hence

$$
\begin{aligned}
\phi\left(u_{n}, \tilde{x}_{n}\right) & =\phi\left(u_{n}, J^{-1}\left(\alpha_{n} J x_{0}+\left(1-\alpha_{n}\right) J z_{n}\right)\right) \\
& =\left\|u_{n}\right\|^{2}-2\left\langle u_{n}, \alpha_{n} J x_{0}+\left(1-\alpha_{n}\right) J z_{n}\right\rangle+\left\|\alpha_{n} J x_{0}+\left(1-\alpha_{n}\right) J z_{n}\right\|^{2} \\
& \leq\left\|u_{n}\right\|^{2}-2 \alpha_{n}\left\langle u_{n}, J x_{0}\right\rangle-2\left(1-\alpha_{n}\right)\left\langle u_{n}, J z_{n}\right\rangle+\alpha_{n}\left\|x_{0}\right\|^{2}+\left(1-\alpha_{n}\right)\left\|z_{n}\right\|^{2} \\
& =\alpha_{n} \phi\left(u_{n}, x_{0}\right)+\left(1-\alpha_{n}\right) \phi\left(u_{n}, z_{n}\right) \\
& \leq \alpha_{n} \phi\left(u_{n}, x_{0}\right)+\phi\left(u_{n}, z_{n}\right) \\
& \leq \alpha_{n} \phi\left(u_{n}, x_{0}\right)+\phi\left(u_{n}, x_{n}\right)+\phi\left(u_{n}, J_{r_{n}} x_{n}\right)
\end{aligned}
$$

Since $\alpha_{n} \rightarrow 0, \phi\left(u_{n}, x_{n}\right) \rightarrow 0$ and $\phi\left(u_{n}, J_{r_{n}} x_{n}\right) \rightarrow 0$, it follows from the boundedness of $\left\{u_{n}\right\}$ that $\phi\left(u_{n}, \tilde{x}_{n}\right) \rightarrow 0$. Thus, in terms of Lemma 2.2, we have that $\left\|u_{n}-\tilde{x}_{n}\right\| \rightarrow 0$ and so $\left\|x_{n}-\tilde{x}_{n}\right\| \rightarrow 0$. Furthermore, it follows from (3.25) that

$$
\begin{aligned}
\phi\left(u, u_{n}\right) & \leq \tilde{\alpha}_{n} \phi\left(u, x_{0}\right)+\left(1-\tilde{\alpha}_{n}\right) \phi\left(u, \tilde{x}_{n}\right)-\left(1-\tilde{\alpha}_{n}\right) \tilde{\beta}_{n}\left(1-\tilde{\beta}_{n}\right) g\left(\left\|J \tilde{x}_{n}-J \tilde{J}_{r_{n}} \tilde{x}_{n}\right\|\right) \\
& \leq \tilde{\alpha}_{n} \phi\left(u, x_{0}\right)+\phi\left(u, \tilde{x}_{n}\right)-\left(1-\tilde{\alpha}_{n}\right) \tilde{\beta}_{n}\left(1-\tilde{\beta}_{n}\right) g\left(\left\|J \tilde{x}_{n}-J \tilde{J}_{r_{n}} \tilde{x}_{n}\right\|\right)
\end{aligned}
$$

and hence

$$
\begin{aligned}
\left(1-\tilde{\alpha}_{n}\right) \tilde{\beta}_{n}\left(1-\tilde{\beta}_{n}\right) g\left(\left\|J \tilde{x}_{n}-J \tilde{J}_{r_{n}} \tilde{x}_{n}\right\|\right) \\
\quad \leq \tilde{\alpha}_{n} \phi\left(u, x_{0}\right)+\phi\left(u, \tilde{x}_{n}\right)-\phi\left(u, u_{n}\right) \\
=\tilde{\alpha}_{n} \phi\left(u, x_{0}\right)+\left\|\tilde{x}_{n}\right\|^{2}-\left\|u_{n}\right\|^{2}+2\left\langle u, J u_{n}-J \tilde{x}_{n}\right\rangle \\
=\tilde{\alpha}_{n} \phi\left(u, x_{0}\right)+\left(\left\|\tilde{x}_{n}\right\|-\left\|u_{n}\right\|\right)\left(\left\|\tilde{x}_{n}\right\|+\left\|u_{n}\right\|\right)+2\left\langle u, J u_{n}-J \tilde{x}_{n}\right\rangle \\
\leq \tilde{\alpha}_{n} \phi\left(u, x_{0}\right)+\left\|\tilde{x}_{n}-u_{n}\right\|\left(\left\|\tilde{x}_{n}\right\|+\left\|u_{n}\right\|\right)+2\|u\|\left\|J u_{n}-J \tilde{x}_{n}\right\| .
\end{aligned}
$$

Since $J$ is uniformly norm-to-norm continuous on bounded subsets of $X$, it follows from $\left\|\tilde{x}_{n}-u_{n}\right\| \rightarrow 0$ that $\left\|J u_{n}-J \tilde{x}_{n}\right\| \rightarrow 0$. Thus from $\tilde{\alpha}_{n} \rightarrow 0, \liminf _{n \rightarrow \infty} \tilde{\beta}_{n}\left(1-\tilde{\beta}_{n}\right)>0$, and the boundedness of both $\left\{\tilde{x}_{n}\right\}$ and $\left\{u_{n}\right\}$, we deduce that $g\left(\left\|J \tilde{x}_{n}-J \tilde{J}_{r_{n}} \widetilde{x}_{n}\right\|\right) \rightarrow 0$. Utilizing the properties of $g$, we have that $\left\|J \tilde{x}_{n}-J \tilde{J}_{r_{n}} \tilde{x}_{n}\right\| \rightarrow 0$. Since $J^{-1}$ is uniformly norm-to-norm continuous on bounded subsets of $X^{*}$, it follows that $\left\|\tilde{x}_{n}-\tilde{J}_{r_{n}} \tilde{x}_{n}\right\| \rightarrow 0$.

Step 5. We claim that $\omega_{w}\left(\left\{x_{n}\right\}\right) \subset T^{-1} 0 \cap \widetilde{T}^{-1} 0 \cap E P$, where

$$
\omega_{w}\left(\left\{x_{n}\right\}\right):=\left\{\widehat{x} \in C: x_{n_{k}} \rightarrow \widehat{x} \text { for some subsequence }\left\{n_{k}\right\} \subset\{n\} \text { with } n_{k} \uparrow \infty\right\} \text {. }
$$

Indeed, since $\left\{x_{n}\right\}$ is bounded and $X$ is reflexive, we know that $\omega_{w}\left(\left\{x_{n}\right\}\right) \neq \emptyset$. Take $\hat{x} \in$ $\omega_{w}\left(\left\{x_{n}\right\}\right)$ arbitrarily. Then there exists a subsequence $\left\{x_{n_{k}}\right\}$ of $\left\{x_{n}\right\}$ such that $x_{n_{k}} \rightarrow \hat{x}$. Hence it follows from $\left\|x_{n}-\tilde{x}_{n}\right\| \rightarrow 0,\left\|x_{n}-J_{r_{n}} x_{n}\right\| \rightarrow 0$, and $\left\|\tilde{x}_{n}-\tilde{J}_{r_{n}} \tilde{x}_{n}\right\| \rightarrow 0$ that $\left\{\tilde{x}_{n_{k}}\right\},\left\{J_{r_{n_{k}}} x_{n_{k}}\right\}$ 
and $\left\{\tilde{J}_{r_{k}} \tilde{x}_{n_{k}}\right\}$ converge weakly to the same point $\widehat{x}$. On the other hand, from (3.28a), (3.28b) and $\lim \inf _{n \rightarrow \infty} r_{n}>0$, we obtain that

$$
\begin{aligned}
& \lim _{n \rightarrow \infty}\left\|A_{r_{n}} x_{n}\right\|=\lim _{n \rightarrow \infty} \frac{1}{r_{n}}\left\|J x_{n}-J J_{r_{n}} x_{n}\right\|=0, \\
& \lim _{n \rightarrow \infty}\left\|\tilde{A}_{r_{n}} \tilde{x}_{n}\right\|=\lim _{n \rightarrow \infty} \frac{1}{r_{n}}\left\|J \tilde{x}_{n}-J \tilde{J}_{r_{n}} \tilde{x}_{n}\right\|=0 .
\end{aligned}
$$

If $z^{*} \in T z$ and $\widetilde{z}^{*} \in \widetilde{T} \widetilde{z}$, then it follows from (2.17) and the monotonicity of the operators $T, \tilde{T}$ that for all $k \geq 1$

$$
\left\langle z-J_{r_{n_{k}}} x_{n_{k}}, z^{*}-A_{r_{n_{k}}} x_{n_{k}}\right\rangle \geq 0, \quad\left\langle\tilde{z}-\tilde{J}_{r_{n_{k}}} \tilde{x}_{n_{k}}, \tilde{z}^{*}-\widetilde{A}_{r_{n_{k}}} \tilde{x}_{n_{k}}\right\rangle \geq 0 .
$$

Letting $k \rightarrow \infty$, we have that $\left\langle z-\widehat{x}, z^{*}\right\rangle \geq 0$ and $\left\langle\tilde{z}-\widehat{x}, \tilde{z}^{*}\right\rangle \geq 0$. Then the maximality of the operators $T, \tilde{T}$ implies that $\hat{x} \in T^{-1} 0$ and $\widehat{x} \in \widetilde{T}^{-1} 0$.

Next, let us show that $\hat{x} \in E P$. Since

$$
\phi\left(u, y_{n}\right) \leq\left(\alpha_{n}+\tilde{\alpha}_{n}-\alpha_{n} \tilde{\alpha}_{n}\right) \phi\left(u, x_{0}\right)+\left(1-\alpha_{n}\right)\left(1-\tilde{\alpha}_{n}\right) \phi\left(u, x_{n}\right),
$$

from $u_{n}=K_{r_{n}} y_{n}$ and Proposition 2.11 it follows that

$$
\begin{aligned}
\phi\left(u_{n}, y_{n}\right)=\phi\left(K_{r_{n}} y_{n}, y_{n}\right) & \leq \phi\left(u, y_{n}\right)-\phi\left(u, K_{r_{n}} y_{n}\right) \\
& \leq\left(\alpha_{n}+\widetilde{\alpha}_{n}-\alpha_{n} \widetilde{\alpha}_{n}\right) \phi\left(u, x_{0}\right)+\left(1-\alpha_{n}\right)\left(1-\widetilde{\alpha}_{n}\right) \phi\left(u, x_{n}\right)-\phi\left(u, K_{r_{n}} y_{n}\right) \\
& \leq\left(\alpha_{n}+\widetilde{\alpha}_{n}-\alpha_{n} \widetilde{\alpha}_{n}\right) \phi\left(u, x_{0}\right)+\phi\left(u, x_{n}\right)-\phi\left(u, u_{n}\right) .
\end{aligned}
$$

Also, since

$$
\begin{aligned}
\left|\varphi\left(u, x_{n}\right)-\varphi\left(u, u_{n}\right)\right| & =\left|\left\|x_{n}\right\|^{2}-\left\|u_{n}\right\|^{2}+2\left\langle u, J u_{n}-J x_{n}\right\rangle\right| \\
& \leq\left|\left\|x_{n}\right\|^{2}-\left\|u_{n}\right\|^{2}\right|+2\left|\left\langle u, J u_{n}-J x_{n}\right\rangle\right| \\
& =\left|\left\|x_{n}\right\|-\left\|u_{n}\right\|\right|\left(\left\|x_{n}\right\|+\left\|u_{n}\right\|\right)+2\|u\|\left\|J u_{n}-J x_{n}\right\| \\
& \leq\left\|x_{n}-u_{n}\right\|\left(\left\|x_{n}\right\|+\left\|u_{n}\right\|\right)+2\|u\|\left\|J u_{n}-J x_{n}\right\|,
\end{aligned}
$$

so we get

$$
\lim _{n \rightarrow \infty}\left(\phi\left(u, x_{n}\right)-\phi\left(u, u_{n}\right)\right)=0
$$

So, from (3.39), $\alpha_{n} \rightarrow 0, \tilde{\alpha}_{n} \rightarrow 0$, and $\phi\left(u, x_{n}\right)-\phi\left(u, u_{n}\right) \rightarrow 0$, we have $\lim _{n \rightarrow \infty} \phi\left(u_{n}, y_{n}\right)=0$. 
Fixed Point Theory and Applications

Since $X$ is uniformly convex and smooth, we conclude from Lemma 2.2 that

$$
\lim _{n \rightarrow \infty}\left\|u_{n}-y_{n}\right\|=0
$$

From $x_{n_{k}} \rightarrow \widehat{x},\left\|x_{n}-u_{n}\right\| \rightarrow 0$, and (3.42), we have $y_{n_{k}} \rightarrow \widehat{x}$ and $u_{n_{k}} \rightarrow \widehat{x}$. we derive

Since $J$ is uniformly norm-to-norm continuous on bounded subsets of $X$, from (3.42)

$$
\lim _{n \rightarrow \infty}\left\|J u_{n}-J y_{n}\right\|=0
$$

From $\lim \inf _{n \rightarrow \infty} r_{n}>0$, it follows that

$$
\lim _{n \rightarrow \infty} \frac{\left\|J u_{n}-J y_{n}\right\|}{r_{n}}=0
$$

By the definition of $u_{n}:=K_{r_{n}} y_{n}$, we have

$$
F\left(u_{n}, y\right)+\frac{1}{r_{n}}\left\langle y-u_{n}, J u_{n}-J y_{n}\right\rangle \geq 0, \quad \forall y \in C,
$$

where

$$
F\left(u_{n}, y\right)=f\left(u_{n}, y\right)+\left\langle A u_{n}, y-u_{n}\right\rangle
$$

Replacing $n$ by $n_{k}$, we have from (A2) that

$$
\frac{1}{r_{n_{k}}}\left\langle y-u_{n_{k}}, J u_{n_{k}}-J y_{n_{k}}\right\rangle \geq-F\left(u_{n_{k}}, y\right) \geq F\left(y, u_{n_{k}}\right), \quad \forall y \in C
$$

Since $y \mapsto f(x, y)+\langle A x, y-x\rangle$ is convex and lower semicontinuous, it is also weakly lower semicontinuous. Letting $n_{k} \rightarrow \infty$ in the last inequality, from (3.44) and (A4), we have

$$
F(y, \widehat{x}) \leq 0, \quad \forall y \in C
$$

For $t$, with $0<t \leq 1$, and $y \in C$, let $y_{t}=t y+(1-t) \widehat{x}$. Since $y \in C$ and $\widehat{x} \in C$, then $y_{t} \in C$ and hence $F\left(y_{t}, \widehat{x}\right) \leq 0$. So, from (A1) we have

$$
0=F\left(y_{t}, y_{t}\right) \leq t F\left(y_{t}, y\right)+(1-t) F\left(y_{t}, \widehat{x}\right) \leq t F\left(y_{t}, y\right)
$$


Dividing by $t$, we have

$$
F\left(y_{t}, y\right) \geq 0, \quad \forall y \in C
$$

Letting $t \downarrow 0$, from (A3) it follows that

$$
F(\widehat{x}, y) \geq 0, \quad \forall y \in C
$$

So, $\widehat{x} \in E P$. Therefore, we obtain that $\omega_{w}\left(\left\{x_{n}\right\}\right) \subset T^{-1} 0 \cap \widetilde{T}^{-1} 0 \cap E P$ by the arbitrariness of $\widehat{x}$.

Step 6 . We claim that $\left\{x_{n}\right\}$ converges strongly to $w=\Pi_{T^{-1} 0 \cap \widetilde{T}^{-1} 0 \cap E P} x_{0}$.

Indeed, from $x_{n+1}=\Pi_{H_{n} \cap W_{n}} x_{0}$ and $w \in T^{-1} 0 \cap \tilde{T}^{-1} 0 \cap E P \subset H_{n} \cap W_{n}$, it follows that

$$
\phi\left(x_{n+1}, x_{0}\right) \leq \phi\left(w, x_{0}\right)
$$

Since the norm is weakly lower semicontinuous, then

$$
\begin{aligned}
\phi\left(\widehat{x}, x_{0}\right) & =\|\widehat{x}\|^{2}-2\left\langle\widehat{x}, J x_{0}\right\rangle+\left\|x_{0}\right\|^{2} \leq \liminf _{k \rightarrow \infty}\left(\left\|x_{n_{k}}\right\|^{2}-2\left\langle x_{n_{k}}, J x_{0}\right\rangle+\left\|x_{0}\right\|^{2}\right) \\
& =\liminf _{k \rightarrow \infty} \phi\left(x_{n_{k}}, x_{0}\right) \leq \limsup _{k \rightarrow \infty} \phi\left(x_{n_{k}}, x_{0}\right) \leq \phi\left(w, x_{0}\right) .
\end{aligned}
$$

From the definition of $\Pi_{T^{-1} 0 \cap \tilde{T}^{-1} 0 \cap E P^{\prime}}$, we have $\widehat{x}=w$. Hence $\lim _{k \rightarrow \infty} \phi\left(x_{n_{k}}, x_{0}\right)=\phi\left(w, x_{0}\right)$, and

$$
\begin{aligned}
0 & =\lim _{k \rightarrow \infty}\left(\phi\left(x_{n_{k}}, x_{0}\right)-\phi\left(w, x_{0}\right)\right)=\lim _{k \rightarrow \infty}\left(\left\|x_{n_{k}}\right\|^{2}-\|w\|^{2}-2\left\langle x_{n_{k}}-w, J x_{0}\right\rangle\right) \\
& =\lim _{k \rightarrow \infty}\left(\left\|x_{n_{k}}\right\|^{2}-\|w\|^{2}\right),
\end{aligned}
$$

which implies that $\lim _{k \rightarrow \infty}\left\|x_{n_{k}}\right\|=\|w\|$. Since $X$ has the Kadec-Klee property, then $x_{n_{k}} \rightarrow$ $w=\Pi_{T^{-1} 0 \cap \tilde{T}^{-1} 0 \cap E P} x_{0}$. Therefore, $\left\{x_{n}\right\}$ converges strongly to $\Pi_{T^{-1} 0 \cap \tilde{T}^{-1} 0 \cap E P} x_{0}$.

Remark 3.2. In Theorem 3.1, let $A \equiv 0, \widetilde{T} \equiv 0$, and $\widetilde{\alpha}_{n}=0, \forall n \geq 0$. Then, for all $\alpha, r \in(0, \infty)$ and $x, y \in C$, we have that

$$
\begin{aligned}
\langle A x-A y, x-y\rangle \geq \alpha\|A x-A y\|^{2}, \\
K_{r}(x)=\left\{u \in C: f(u, y)+\langle A u, y-u\rangle+\frac{1}{r}\langle y-u, J u-J x\rangle \geq 0, \forall y \in C\right\} \\
=\left\{u \in C: f(u, y)+\frac{1}{r}\langle y-u, J u-J x\rangle \geq 0, \forall y \in C\right\}=T_{r}(x) .
\end{aligned}
$$


Fixed Point Theory and Applications

Moreover, there hold the following

$$
\begin{aligned}
H_{n} & =\left\{z \in C: \phi\left(z, K_{r_{n}} y_{n}\right) \leq\left(\alpha_{n}+\tilde{\alpha}_{n}-\alpha_{n} \tilde{\alpha}_{n}\right) \phi\left(z, x_{0}\right)+\left(1-\alpha_{n}\right)\left(1-\tilde{\alpha}_{n}\right) \phi\left(z, x_{n}\right)\right\} \\
& =\left\{z \in C: \phi\left(z, T_{r_{n}} y_{n}\right) \leq \alpha_{n} \phi\left(z, x_{0}\right)+\left(1-\alpha_{n}\right) \phi\left(z, x_{n}\right)\right\}, \\
y_{n} & =J^{-1}\left(\tilde{\alpha}_{n} J x_{0}+\left(1-\tilde{\alpha}_{n}\right)\left(\tilde{\beta}_{n} J \tilde{x}_{n}+\left(1-\tilde{\beta}_{n}\right) J \tilde{J}_{r_{n}} \tilde{x}_{n}\right)\right) \\
& =J^{-1}\left(\tilde{\beta}_{n} J \tilde{x}_{n}+\left(1-\tilde{\beta}_{n}\right) J \tilde{J}_{r_{n}} \tilde{x}_{n}\right) \\
& =J^{-1}\left(\tilde{\beta}_{n} J \tilde{x}_{n}+\left(1-\tilde{\beta}_{n}\right) J \tilde{x}_{n}\right) \\
& =J^{-1} J \tilde{x}_{n}=\tilde{x}_{n}
\end{aligned}
$$

and hence

$$
y_{n}=\tilde{x}_{n}=J^{-1}\left(\alpha_{n} J x_{0}+\left(1-\alpha_{n}\right)\left(\beta_{n} J x_{n}+\left(1-\beta_{n}\right) J J_{r_{n}} x_{n}\right)\right) .
$$

In this case, Theorem 3.1 reduces to [17, Theorem 3.1].

\section{Weak Convergence Theorem}

In this section, we present the following algorithm for finding a common element of the solution set of a generalized equilibrium problem and the set $T^{-1} 0 \cap \widetilde{T}^{-1} 0$ for two maximal monotone operators $T$ and $\widetilde{T}$.

Let $x_{0} \in X$ be chosen arbitrarily and consider the sequence $\left\{x_{n}\right\}$ generated by

$$
\begin{gathered}
\tilde{x}_{n}=J^{-1}\left(\alpha_{n} J x_{0}+\left(1-\alpha_{n}\right)\left(\beta_{n} J K_{r_{n}} x_{n}+\left(1-\beta_{n}\right) J J_{r_{n}} K_{r_{n}} x_{n}\right)\right), \\
x_{n+1}=J^{-1}\left(\widetilde{\alpha}_{n} J x_{0}+\left(1-\widetilde{\alpha}_{n}\right)\left(\tilde{\beta}_{n} J K_{r_{n}} \tilde{x}_{n}+\left(1-\widetilde{\beta}_{n}\right) J \widetilde{J}_{r_{n}} K_{r_{n}} \widetilde{x}_{n}\right)\right), \quad n=0,1,2, \ldots,
\end{gathered}
$$

where $\left\{\alpha_{n}\right\},\left\{\beta_{n}\right\},\left\{\widetilde{\alpha}_{n}\right\},\left\{\tilde{\beta}_{n}\right\} \subset[0,1],\left\{r_{n}\right\} \subset(0, \infty)$, and $K_{r}, r>0$ is defined by (2.14).

Before proving a weak convergence theorem, we need the following proposition.

Proposition 4.1. Suppose that Assumption 2.1 is fulfilled and let $\left\{x_{n}\right\}$ be a sequence defined by (4.1), where $\left\{\alpha_{n}\right\},\left\{\beta_{n}\right\},\left\{\tilde{\alpha}_{n}\right\},\left\{\tilde{\beta}_{n}\right\} \subset[0,1]$ satisfy the following conditions:

$$
\sum_{n=0}^{\infty} \alpha_{n}<\infty, \quad \sum_{n=0}^{\infty} \widetilde{\alpha}_{n}<\infty, \quad \liminf _{n \rightarrow \infty} \beta_{n}\left(1-\beta_{n}\right)>0, \quad \liminf _{n \rightarrow \infty} \tilde{\beta}_{n}\left(1-\tilde{\beta}_{n}\right)>0 .
$$

Then, $\left\{\Pi_{T^{-1} 0 \cap \tilde{T}^{-1} 0 \cap E P} x_{n}\right\}$ converges strongly to $z \in T^{-1} 0 \cap \widetilde{T}^{-1} 0 \cap E P$, where $\Pi_{T^{-1} O \cap \widetilde{T}^{-1} 0 \cap E P}$ is the generalized projection of $X$ onto $T^{-1} 0 \cap \widetilde{T}^{-1} 0 \cap E P$. 
Proof. We set $\Omega:=T^{-1} 0 \cap \widetilde{T}^{-1} 0 \cap E P$ and

$$
\begin{aligned}
& u_{n}:=K_{r_{n}} x_{n}, \quad y_{n}:=J^{-1}\left(\beta_{n} J u_{n}+\left(1-\beta_{n}\right) J J_{r_{n}} u_{n}\right), \\
& \widetilde{u}_{n}:=K_{r_{n}} \tilde{x}_{n}, \quad \tilde{y}_{n}:=J^{-1}\left(\widetilde{\beta}_{n} J \widetilde{u}_{n}+\left(1-\widetilde{\beta}_{n}\right) J \widetilde{J}_{r_{n}} \widetilde{u}_{n}\right),
\end{aligned}
$$

so that

$$
\begin{gathered}
\tilde{x}_{n}=J^{-1}\left(\alpha_{n} J x_{0}+\left(1-\alpha_{n}\right) J y_{n}\right), \\
x_{n+1}=J^{-1}\left(\tilde{\alpha}_{n} J x_{0}+\left(1-\tilde{\alpha}_{n}\right) J \tilde{y}_{n}\right), \quad n=0,1,2, \ldots
\end{gathered}
$$

Then, in terms of Lemma 2.5 and Proposition 2.11, $\Omega$ is a nonempty closed convex subset of $X$ such that $\Omega \subset C$. We first prove that $\left\{x_{n}\right\}$ is bounded. Fix $u \in \Omega$. Note that by the first and third of (4.3), $u_{n}, \tilde{u}_{n} \in C$ and

$$
\begin{aligned}
& F\left(u_{n}, y\right)+\frac{1}{r_{n}}\left\langle y-u_{n}, J u_{n}-J x_{n}\right\rangle \geq 0, \quad \forall y \in C, \\
& F\left(\tilde{u}_{n}, y\right)+\frac{1}{r_{n}}\left\langle y-\tilde{u}_{n}, J \tilde{u}_{n}-J \tilde{x}_{n}\right\rangle \geq 0, \quad \forall y \in C .
\end{aligned}
$$

Here, each $K_{r_{n}}$ is relatively nonexpansive. Then from Proposition 2.11, we obtain

$$
\begin{aligned}
\phi\left(u, y_{n}\right) & =\phi\left(u, J^{-1}\left(\beta_{n} J u_{n}+\left(1-\beta_{n}\right) J J_{r_{n}} u_{n}\right)\right) \\
& =\|u\|^{2}-2\left\langle u, \beta_{n} J u_{n}+\left(1-\beta_{n}\right) J J_{r_{n}} u_{n}\right\rangle+\left\|\beta_{n} J u_{n}+\left(1-\beta_{n}\right) J J_{r_{n}} u_{n}\right\|^{2} \\
& \leq\|u\|^{2}-2 \beta_{n}\left\langle u, J u_{n}\right\rangle-2\left(1-\beta_{n}\right)\left\langle u, J J_{r_{n}} u_{n}\right\rangle+\beta_{n}\left\|u_{n}\right\|^{2}+\left(1-\beta_{n}\right)\left\|J_{r_{n}} u_{n}\right\|^{2} \\
& =\beta_{n} \phi\left(u, u_{n}\right)+\left(1-\beta_{n}\right) \phi\left(u, J_{r_{n}} u_{n}\right) \\
& \leq \beta_{n} \phi\left(u, u_{n}\right)+\left(1-\beta_{n}\right) \phi\left(u, u_{n}\right) \\
& =\phi\left(u, u_{n}\right)=\phi\left(u, K_{r_{n}} x_{n}\right) \leq \phi\left(u, x_{n}\right), \\
\phi\left(u, \tilde{y}_{n}\right) & =\phi\left(u, J^{-1}\left(\tilde{\beta}_{n} J \tilde{u}_{n}+\left(1-\tilde{\beta}_{n}\right) J \tilde{J}_{r_{n}} \tilde{u}_{n}\right)\right) \\
& =\|u\|^{2}-2\left\langle u, \tilde{\beta}_{n} J \tilde{u}_{n}+\left(1-\tilde{\beta}_{n}\right) J \tilde{J}_{r_{n}} \tilde{u}_{n}\right\rangle+\left\|\tilde{\beta}_{n} J \tilde{u}_{n}+\left(1-\tilde{\beta}_{n}\right) J \tilde{J}_{r_{n}} \tilde{u}_{n}\right\|^{2} \\
& \leq\|u\|^{2}-2 \tilde{\beta}_{n}\left\langle u, J \tilde{u}_{n}\right\rangle-2\left(1-\tilde{\beta}_{n}\right)\left\langle u, J \tilde{J}_{r_{n}} \tilde{u}_{n}\right\rangle+\tilde{\beta}_{n}\left\|\tilde{u}_{n}\right\|^{2}+\left(1-\tilde{\beta}_{n}\right)\left\|\tilde{J}_{r_{n}} \tilde{u}_{n}\right\|^{2} \\
& =\tilde{\beta}_{n} \phi\left(u, \tilde{u}_{n}\right)+\left(1-\tilde{\beta}_{n}\right) \phi\left(u, \tilde{J}_{r_{n}} \tilde{u}_{n}\right) \\
& \leq \tilde{\beta}_{n} \phi\left(u, \tilde{u}_{n}\right)+\left(1-\tilde{\beta}_{n}\right) \phi\left(u, \tilde{u}_{n}\right) \\
& =\phi\left(u, \tilde{u}_{n}\right)=\phi\left(u, K_{r_{n}} \tilde{x}_{n}\right) \leq \phi\left(u, \tilde{x}_{n}\right),
\end{aligned}
$$


and hence by Proposition 2.11

$$
\begin{aligned}
\phi\left(u, \tilde{x}_{n}\right) & =\phi\left(u, J^{-1}\left(\alpha_{n} J x_{0}+\left(1-\alpha_{n}\right) J y_{n}\right)\right) \\
& =\|u\|^{2}-2\left\langle u, \alpha_{n} J x_{0}+\left(1-\alpha_{n}\right) J y_{n}\right\rangle+\left\|\alpha_{n} J x_{0}+\left(1-\alpha_{n}\right) J y_{n}\right\|^{2} \\
& \leq\|u\|^{2}-2 \alpha_{n}\left\langle u, J x_{0}\right\rangle-2\left(1-\alpha_{n}\right)\left\langle u, J y_{n}\right\rangle+\alpha_{n}\left\|x_{0}\right\|^{2}+\left(1-\alpha_{n}\right)\left\|y_{n}\right\|^{2} \\
& =\alpha_{n} \phi\left(u, x_{0}\right)+\left(1-\alpha_{n}\right) \phi\left(u, y_{n}\right) \\
& \leq \alpha_{n} \phi\left(u, x_{0}\right)+\phi\left(u, y_{n}\right) \\
& \leq \phi\left(u, x_{n}\right)+\alpha_{n} \phi\left(u, x_{0}\right), \\
\phi\left(u, x_{n+1}\right) & =\phi\left(u, J^{-1}\left(\tilde{\alpha}_{n} J x_{0}+\left(1-\tilde{\alpha}_{n}\right) J \tilde{y}_{n}\right)\right) \\
& =\|u\|^{2}-2\left\langle u, \tilde{\alpha}_{n} J x_{0}+\left(1-\tilde{\alpha}_{n}\right) J \tilde{y}_{n}\right\rangle+\left\|\tilde{\alpha}_{n} J x_{0}+\left(1-\tilde{\alpha}_{n}\right) J \tilde{y}_{n}\right\|^{2} \\
& \leq\|u\|^{2}-2 \tilde{\alpha}_{n}\left\langle u, J x_{0}\right\rangle-2\left(1-\tilde{\alpha}_{n}\right)\left\langle u, J \tilde{y}_{n}\right\rangle+\tilde{\alpha}_{n}\left\|x_{0}\right\|^{2}+\left(1-\tilde{\alpha}_{n}\right)\left\|\tilde{y}_{n}\right\|^{2} \\
& =\tilde{\alpha}_{n} \phi\left(u, x_{0}\right)+\left(1-\tilde{\alpha}_{n}\right) \phi\left(u, \tilde{y}_{n}\right) \\
& \leq \tilde{\alpha}_{n} \phi\left(u, x_{0}\right)+\phi\left(u, \tilde{y}_{n}\right) \\
& \leq \phi\left(u, \tilde{x}_{n}\right)+\tilde{\alpha}_{n} \phi\left(u, x_{0}\right) .
\end{aligned}
$$

Consequently, the last two inequalities yield that

$$
\begin{aligned}
\phi\left(u, x_{n+1}\right) & \leq \phi\left(u, \tilde{x}_{n}\right)+\tilde{\alpha}_{n} \phi\left(u, x_{0}\right) \\
& \leq \phi\left(u, x_{n}\right)+\alpha_{n} \phi\left(u, x_{0}\right)+\tilde{\alpha}_{n} \phi\left(u, x_{0}\right) \\
& =\phi\left(u, x_{n}\right)+\left(\alpha_{n}+\tilde{\alpha}_{n}\right) \phi\left(u, x_{0}\right)
\end{aligned}
$$

for all $n \geq 0$. So, from $\sum_{n=0}^{\infty} \alpha_{n}<\infty, \sum_{n=0}^{\infty} \widetilde{\alpha}_{n}<\infty$, and Lemma 2.13, we deduce that $\lim _{n \rightarrow \infty} \phi\left(u, x_{n}\right)$ exists. This implies that $\left\{\phi\left(u, x_{n}\right)\right\}$ is bounded. Thus, $\left\{x_{n}\right\}$ is bounded and so are $\left\{u_{n}\right\},\left\{\tilde{u}_{n}\right\},\left\{J_{r_{n}} u_{n}\right\}$, and $\left\{\widetilde{J}_{r_{n}} \widetilde{u}_{n}\right\}$.

Define $z_{n}=\Pi_{\Omega} x_{n}$ for all $n \geq 0$. Let us show that $\left\{z_{n}\right\}$ is bounded. Indeed, observe that

$$
\begin{aligned}
\left(\left\|z_{n}\right\|-\left\|x_{n}\right\|\right)^{2} & \leq \phi\left(z_{n}, x_{n}\right)=\phi\left(\Pi_{\Omega} x_{n}, x_{n}\right) \leq \phi\left(p, x_{n}\right)-\phi\left(p, \Pi_{\Omega} x_{n}\right) \\
& =\phi\left(p, x_{n}\right)-\phi\left(p, z_{n}\right) \leq \phi\left(p, x_{n}\right)
\end{aligned}
$$

for each $p \in \Omega$. This, together with the boundedness of $\left\{x_{n}\right\}$, implies that $\left\{z_{n}\right\}$ is bounded and so is $\phi\left(z_{n}, x_{0}\right)$. Furthermore, from $z_{n} \in \Omega$ and (4.6e), we have

$$
\phi\left(z_{n}, x_{n+1}\right) \leq \phi\left(z_{n}, x_{n}\right)+\left(\alpha_{n}+\tilde{\alpha}_{n}\right) \phi\left(z_{n}, x_{0}\right) .
$$


Since $\Pi_{\Omega}$ is the generalized projection, then, from Lemma 2.4 we obtain

$$
\begin{aligned}
\phi\left(z_{n+1}, x_{n+1}\right) & =\phi\left(\Pi_{\Omega} x_{n+1}, x_{n+1}\right) \leq \phi\left(z_{n}, x_{n+1}\right)-\phi\left(z_{n}, \Pi_{\Omega} x_{n+1}\right) \\
& =\phi\left(z_{n}, x_{n+1}\right)-\phi\left(z_{n}, z_{n+1}\right) \leq \phi\left(z_{n}, x_{n+1}\right) .
\end{aligned}
$$

Hence, from (4.8), it follows that $\phi\left(z_{n+1}, x_{n+1}\right) \leq \phi\left(z_{n}, x_{n}\right)+\left(\alpha_{n}+\tilde{\alpha}_{n}\right) \phi\left(z_{n}, x_{0}\right)$.

Note that $\sum_{n=0}^{\infty} \alpha_{n}<\infty, \sum_{n=0}^{\infty} \widetilde{\alpha}_{n}<\infty$, and $\left\{\phi\left(z_{n}, x_{0}\right)\right\}$ is bounded, so that $\sum_{n=0}^{\infty}\left(\alpha_{n}+\right.$ $\left.\tilde{\alpha}_{n}\right) \phi\left(z_{n}, x_{0}\right)<\infty$. Therefore, $\left\{\phi\left(z_{n}, x_{n}\right)\right\}$ is a convergent sequence. On the other hand, from (4.6e) we derive, for all $m \geq 0$,

$$
\phi\left(u, x_{n+m}\right) \leq \phi\left(u, x_{n}\right)+\sum_{j=0}^{m-1}\left(\alpha_{n+j}+\tilde{\alpha}_{n+j}\right) \phi\left(u, x_{0}\right) .
$$

In particular, we have

$$
\phi\left(z_{n}, x_{n+m}\right) \leq \phi\left(z_{n}, x_{n}\right)+\sum_{j=0}^{m-1}\left(\alpha_{n+j}+\widetilde{\alpha}_{n+j}\right) \phi\left(z_{n}, x_{0}\right) .
$$

Consequently, from $z_{n+m}=\prod_{\Omega} x_{n+m}$ and Lemma 2.4, we have

$$
\phi\left(z_{n}, z_{n+m}\right)+\phi\left(z_{n+m}, x_{n+m}\right) \leq \phi\left(z_{n}, x_{n+m}\right) \leq \phi\left(z_{n}, x_{n}\right)+\sum_{j=0}^{m-1}\left(\alpha_{n+j}+\tilde{\alpha}_{n+j}\right) \phi\left(z_{n}, x_{0}\right)
$$

and hence

$$
\phi\left(z_{n}, z_{n+m}\right) \leq \phi\left(z_{n}, x_{n}\right)-\phi\left(z_{n+m}, x_{n+m}\right)+\sum_{j=0}^{m-1}\left(\alpha_{n+j}+\tilde{\alpha}_{n+j}\right) \phi\left(z_{n}, x_{0}\right) .
$$

Let $r=\sup \left\{\left\|z_{n}\right\|: n \geq 0\right\}$. From Lemma 2.7, there exists a continuous, strictly increasing, and convex function $g$ with $g(0)=0$ such that

$$
g(\|x-y\|) \leq \phi(x, y), \quad \forall x, y \in B_{r} .
$$

So, we have

$$
\begin{aligned}
g\left(\left\|z_{n}-z_{n+m}\right\|\right) & \leq \phi\left(z_{n}, z_{n+m}\right) \\
& \leq \phi\left(z_{n}, x_{n}\right)-\phi\left(z_{n+m}, x_{n+m}\right)+\sum_{j=0}^{m-1}\left(\alpha_{n+j}+\widetilde{\alpha}_{n+j}\right) \phi\left(z_{n}, x_{0}\right) .
\end{aligned}
$$

Since $\left\{\phi\left(z_{n}, x_{n}\right)\right\}$ is a convergent sequence, $\left\{\phi\left(z_{n}, x_{0}\right)\right\}$ is bounded and $\sum_{n=0}^{\infty}\left(\alpha_{n}+\widetilde{\alpha}_{n}\right)$ is convergent; from the property of $g$, we have that $\left\{z_{n}\right\}$ is a Cauchy sequence. Since $\Omega$ is closed, $\left\{z_{n}\right\}$ converges strongly to $z \in \Omega$. This completes the proof. 
Fixed Point Theory and Applications

Now, we are in a position to prove the following theorem.

Theorem 4.2. Suppose that Assumption 2.1 is fulfilled and let $\left\{x_{n}\right\}$ be a sequence defined by (4.1), where $\left\{\alpha_{n}\right\},\left\{\beta_{n}\right\},\left\{\tilde{\alpha}_{n}\right\},\left\{\tilde{\beta}_{n}\right\} \subset[0,1]$ satisfy the following conditions:

$$
\sum_{n=0}^{\infty} \alpha_{n}<\infty, \quad \sum_{n=0}^{\infty} \widetilde{\alpha}_{n}<\infty, \quad \liminf _{n \rightarrow \infty} \beta_{n}\left(1-\beta_{n}\right)>0, \quad \liminf _{n \rightarrow \infty} \tilde{\beta}_{n}\left(1-\tilde{\beta}_{n}\right)>0,
$$

and $\left\{r_{n}\right\} \subset(0, \infty)$ satisfies $\liminf _{n \rightarrow \infty} r_{n}>0$. If $J$ is weakly sequentially continuous, then $\left\{x_{n}\right\}$ converges weakly to $z \in T^{-1} 0 \cap \widetilde{T}^{-1} 0 \cap E P$, where $z=\lim _{n \rightarrow \infty} \Pi_{T^{-1} 0 \cap \tilde{T}^{-1} 0 \cap E P} x_{n}$.

Proof. We consider the notations (4.3). As in the proof of Proposition 4.1, we have that $\left\{x_{n}\right\},\left\{u_{n}\right\},\left\{J_{r_{n}} u_{n}\right\},\left\{\tilde{x}_{n}\right\},\left\{\tilde{u}_{n}\right\}$, and $\left\{\tilde{J}_{r_{n}} \tilde{u}_{n}\right\}$ are bounded sequences. Let

$$
r=\sup \left\{\left\|u_{n}\right\|,\left\|J_{r_{n}} u_{n}\right\|,\left\|\tilde{u}_{n}\right\|,\left\|\tilde{J}_{r_{n}} \tilde{u}_{n}\right\|: n \geq 0\right\}
$$

From Lemma 2.6 and as in the proof of Theorem 3.1, there exists a continuous, strictly increasing, and convex function $g$ with $g(0)=0$ such that

$$
\left\|\alpha x^{*}+(1-\alpha) y^{*}\right\|^{2} \leq \alpha\left\|x^{*}\right\|^{2}+(1-\alpha)\left\|y^{*}\right\|^{2}-\alpha(1-\alpha) g\left(\left\|x^{*}-y^{*}\right\|\right)
$$

for $x^{*}, y^{*} \in B_{r}^{*}$ and $\alpha \in[0,1]$. Observe that for $u \in \Omega:=T^{-1} 0 \cap \widetilde{T}^{-1} 0 \cap E P$,

$$
\begin{aligned}
\phi\left(u, y_{\mathrm{n}}\right)= & \phi\left(u, J^{-1}\left(\beta_{n} J u_{n}+\left(1-\beta_{n}\right) J J_{r_{n}} u_{n}\right)\right) \\
= & \|u\|^{2}-2\left\langle u, \beta_{n} J u_{n}+\left(1-\beta_{n}\right) J J_{r_{n}} u_{n}\right\rangle+\left\|\beta_{n} J u_{n}+\left(1-\beta_{n}\right) J J_{r_{n}} u_{n}\right\|^{2} \\
\leq & \|u\|^{2}-2 \beta_{n}\left\langle u, J u_{n}\right\rangle-2\left(1-\beta_{n}\right)\left\langle u, J J_{r_{n}} u_{n}\right\rangle \\
& +\beta_{n}\left\|u_{n}\right\|^{2}+\left(1-\beta_{n}\right)\left\|J_{r_{n}} u_{n}\right\|^{2}-\beta_{n}\left(1-\beta_{n}\right) g\left(\left\|J u_{n}-J J_{r_{n}} u_{n}\right\|\right) \\
\leq & \beta_{n} \phi\left(u, u_{n}\right)+\left(1-\beta_{n}\right) \phi\left(u, J_{r_{n}} u_{n}\right)-\beta_{n}\left(1-\beta_{n}\right) g\left(\left\|J u_{n}-J J_{r_{n}} u_{n}\right\|\right) \\
\leq & \beta_{n} \phi\left(u, u_{n}\right)+\left(1-\beta_{n}\right) \phi\left(u, u_{n}\right)-\beta_{n}\left(1-\beta_{n}\right) g\left(\left\|J u_{n}-J J_{r_{n}} u_{n}\right\|\right) \\
= & \phi\left(u, u_{n}\right)-\beta_{n}\left(1-\beta_{n}\right) g\left(\left\|J u_{n}-J J_{r_{n}} u_{n}\right\|\right),
\end{aligned}
$$




$$
\begin{aligned}
\phi\left(u, \tilde{y}_{n}\right)= & \phi\left(u, J^{-1}\left(\tilde{\beta}_{n} J \tilde{u}_{n}+\left(1-\tilde{\beta}_{n}\right) J \tilde{J}_{r_{n}} \tilde{u}_{n}\right)\right) \\
= & \|u\|^{2}-2\left\langle u, \tilde{\beta}_{n} J \tilde{u}_{n}+\left(1-\tilde{\beta}_{n}\right) J \tilde{J}_{r_{n}} \tilde{u}_{n}\right\rangle+\left\|\tilde{\beta}_{n} J \tilde{u}_{n}+\left(1-\tilde{\beta}_{n}\right) J \tilde{J}_{r_{n}} \tilde{u}_{n}\right\|^{2} \\
\leq & \|u\|^{2}-2 \tilde{\beta}_{n}\left\langle u, J \tilde{u}_{n}\right\rangle-2\left(1-\tilde{\beta}_{n}\right)\left\langle u, J \tilde{J}_{r_{n}} \tilde{u}_{n}\right\rangle \\
& +\tilde{\beta}_{n}\left\|\tilde{u}_{n}\right\|^{2}+\left(1-\tilde{\beta}_{n}\right)\left\|\tilde{J}_{r_{n}} \tilde{u}_{n}\right\|^{2}-\tilde{\beta}_{n}\left(1-\tilde{\beta}_{n}\right) g\left(\left\|J \tilde{u}_{n}-J \tilde{J}_{r_{n}} \tilde{u}_{n}\right\|\right) \\
\leq & \tilde{\beta}_{n} \phi\left(u, \tilde{u}_{n}\right)+\left(1-\tilde{\beta}_{n}\right) \phi\left(u, \tilde{J}_{r_{n}} \tilde{u}_{n}\right)-\tilde{\beta}_{n}\left(1-\tilde{\beta}_{n}\right) g\left(\left\|J \tilde{u}_{n}-J \tilde{J}_{r_{n}} \tilde{u}_{n}\right\|\right) \\
\leq & \tilde{\beta}_{n} \phi\left(u, \tilde{u}_{n}\right)+\left(1-\tilde{\beta}_{n}\right) \phi\left(u, \tilde{u}_{n}\right)-\tilde{\beta}_{n}\left(1-\tilde{\beta}_{n}\right) g\left(\left\|J \tilde{u}_{n}-J \tilde{J}_{r_{n}} \tilde{u}_{n}\right\|\right) \\
= & \phi\left(u, \tilde{u}_{n}\right)-\tilde{\beta}_{n}\left(1-\tilde{\beta}_{n}\right) g\left(\left\|J \tilde{u}_{n}-J \tilde{J}_{r_{n}} \tilde{u}_{n}\right\|\right) .
\end{aligned}
$$

Hence,

$$
\begin{aligned}
& \phi\left(u, \tilde{x}_{n}\right)=\phi\left(u, J^{-1}\left(\alpha_{n} J x_{0}+\left(1-\alpha_{n}\right) J y_{n}\right)\right) \\
& =\|u\|^{2}-2\left\langle u, \alpha_{n} J x_{0}+\left(1-\alpha_{n}\right) J y_{n}\right\rangle+\left\|\alpha_{n} J x_{0}+\left(1-\alpha_{n}\right) J y_{n}\right\|^{2} \\
& \leq\|u\|^{2}-2 \alpha_{n}\left\langle u, J x_{0}\right\rangle-2\left(1-\alpha_{n}\right)\left\langle u, J y_{n}\right\rangle+\alpha_{n}\left\|x_{0}\right\|^{2}+\left(1-\alpha_{n}\right)\left\|y_{n}\right\|^{2} \\
& =\alpha_{n} \phi\left(u, x_{0}\right)+\left(1-\alpha_{n}\right) \phi\left(u, y_{n}\right) \\
& \leq \alpha_{n} \phi\left(u, x_{0}\right)+\phi\left(u, y_{n}\right) \\
& \leq \alpha_{n} \phi\left(u, x_{0}\right)+\phi\left(u, u_{n}\right)-\beta_{n}\left(1-\beta_{n}\right) g\left(\left\|J u_{n}-J J_{r_{n}} u_{n}\right\|\right) \\
& =\alpha_{n} \phi\left(u, x_{0}\right)+\phi\left(u, K_{r_{n}} x_{n}\right)-\beta_{n}\left(1-\beta_{n}\right) g\left(\left\|J u_{n}-J J_{r_{n}} u_{n}\right\|\right) \\
& \leq \alpha_{n} \phi\left(u, x_{0}\right)+\phi\left(u, x_{n}\right)-\beta_{n}\left(1-\beta_{n}\right) g\left(\left\|J u_{n}-J J_{r_{n}} u_{n}\right\|\right), \\
& \phi\left(u, x_{n+1}\right)=\phi\left(u, J^{-1}\left(\tilde{\alpha}_{n} J x_{0}+\left(1-\tilde{\alpha}_{n}\right) J \tilde{y}_{n}\right)\right) \\
& =\|u\|^{2}-2\left\langle u, \tilde{\alpha}_{n} J x_{0}+\left(1-\tilde{\alpha}_{n}\right) J \tilde{y}_{n}\right\rangle+\left\|\widetilde{\alpha}_{n} J x_{0}+\left(1-\tilde{\alpha}_{n}\right) J \tilde{y}_{n}\right\|^{2} \\
& \leq\|u\|^{2}-2 \widetilde{\alpha}_{n}\left\langle u, J x_{0}\right\rangle-2\left(1-\widetilde{\alpha}_{n}\right)\left\langle u, J \tilde{y}_{n}\right\rangle+\widetilde{\alpha}_{n}\left\|x_{0}\right\|^{2}+\left(1-\widetilde{\alpha}_{n}\right)\left\|\tilde{y}_{n}\right\|^{2} \\
& =\tilde{\alpha}_{n} \phi\left(u, x_{0}\right)+\left(1-\tilde{\alpha}_{n}\right) \phi\left(u, \tilde{y}_{n}\right) \\
& \leq \tilde{\alpha}_{n} \phi\left(u, x_{0}\right)+\phi\left(u, \tilde{y}_{n}\right) \\
& \leq \tilde{\alpha}_{n} \phi\left(u, x_{0}\right)+\phi\left(u, \tilde{u}_{n}\right)-\tilde{\beta}_{n}\left(1-\tilde{\beta}_{n}\right) g\left(\left\|J \tilde{u}_{n}-J \tilde{J}_{r_{n}} \tilde{u}_{n}\right\|\right) \\
& =\tilde{\alpha}_{\mathrm{n}} \phi\left(u, x_{0}\right)+\phi\left(u, K_{r_{n}} \widetilde{x}_{n}\right)-\tilde{\beta}_{n}\left(1-\widetilde{\beta}_{n}\right) g\left(\left\|J \tilde{u}_{n}-J \tilde{J}_{r_{n}} \tilde{u}_{n}\right\|\right) \\
& \left.\leq \tilde{\alpha}_{n} \phi\left(u, x_{0}\right)+\phi\left(u, \tilde{x}_{n}\right)-\tilde{\beta}_{n}\left(1-\tilde{\beta}_{n}\right) g\left(\left\|J \tilde{u}_{n}-J \widetilde{J}_{r_{n}} \tilde{u}_{n}\right\|\right) \cdot\right)
\end{aligned}
$$


Consequently, the last two inequalities yield that

$$
\begin{aligned}
\phi\left(u, x_{n+1}\right) \leq & \tilde{\alpha}_{n} \phi\left(u, x_{0}\right)+\phi\left(u, \tilde{x}_{n}\right)-\tilde{\beta}_{n}\left(1-\tilde{\beta}_{n}\right) g\left(\left\|J \tilde{u}_{n}-J \tilde{J}_{r_{n}} \tilde{u}_{n}\right\|\right) \\
\leq & \tilde{\alpha}_{n} \phi\left(u, x_{0}\right)+\alpha_{n} \phi\left(u, x_{0}\right)+\phi\left(u, x_{n}\right)-\beta_{n}\left(1-\beta_{n}\right) g\left(\left\|J u_{n}-J J_{r_{n}} u_{n}\right\|\right) \\
& -\tilde{\beta}_{n}\left(1-\tilde{\beta}_{n}\right) g\left(\left\|J \tilde{u}_{n}-J \widetilde{J}_{r_{n}} \tilde{u}_{n}\right\|\right) \\
= & \phi\left(u, x_{n}\right)+\left(\alpha_{n}+\tilde{\alpha}_{n}\right) \phi\left(u, x_{0}\right)-\beta_{n}\left(1-\beta_{n}\right) g\left(\left\|J u_{n}-J J_{r_{n}} u_{n}\right\|\right) \\
& -\tilde{\beta}_{n}\left(1-\tilde{\beta}_{n}\right) g\left(\left\|J \tilde{u}_{n}-J \tilde{J}_{r_{n}} \tilde{u}_{n}\right\|\right) .
\end{aligned}
$$

Thus, we have

$$
\begin{gathered}
\beta_{n}\left(1-\beta_{n}\right) g\left(\left\|J u_{n}-J J_{r_{n}} u_{n}\right\|\right)+\tilde{\beta}_{n}\left(1-\tilde{\beta}_{n}\right) g\left(\left\|J \tilde{u}_{n}-J \tilde{J}_{r_{n}} \tilde{u}_{n}\right\|\right) \\
\leq \phi\left(u, x_{n}\right)-\phi\left(u, x_{n+1}\right)+\left(\alpha_{n}+\tilde{\alpha}_{n}\right) \phi\left(u, x_{0}\right) .
\end{gathered}
$$

By the proof of Proposition 4.1, it is known that $\left\{\phi\left(u, x_{n}\right)\right\}$ is convergent; since $\lim _{n \rightarrow \infty} \alpha_{n}=0$, $\lim _{n \rightarrow \infty} \tilde{\alpha}_{n}=0, \liminf _{n \rightarrow \infty} \beta_{n}\left(1-\beta_{n}\right)>0$, and $\liminf _{n \rightarrow \infty} \widetilde{\beta}_{n}\left(1-\tilde{\beta}_{n}\right)>0$, then we have

$$
\lim _{n \rightarrow \infty} g\left(\left\|J u_{n}-J J_{r_{n}} u_{n}\right\|\right)=\lim _{n \rightarrow \infty} g\left(\left\|J \tilde{u}_{n}-J \tilde{J}_{r_{n}} \tilde{u}_{n}\right\|\right)=0
$$

Taking into account the properties of $g$, as in the proof of Theorem 3.1, we have

$$
\begin{aligned}
& \lim _{n \rightarrow \infty}\left\|J u_{n}-J J_{r_{n}} u_{n}\right\|=\lim _{n \rightarrow \infty}\left\|u_{n}-J_{r_{n}} u_{n}\right\|=0, \\
& \lim _{n \rightarrow \infty}\left\|J \tilde{u}_{n}-J \tilde{J}_{r_{n}} \tilde{u}_{n}\right\|=\lim _{n \rightarrow \infty}\left\|\tilde{u}_{n}-\widetilde{J}_{r_{n}} \tilde{u}_{n}\right\|=0,
\end{aligned}
$$

since $J^{-1}$ is uniformly norm-to-norm continuous on bounded subsets of $X^{*}$.

Now let us show that

$$
\lim _{n \rightarrow \infty} \phi\left(u, x_{n}\right)=\lim _{n \rightarrow \infty} \phi\left(u, \tilde{x}_{n}\right)=\lim _{n \rightarrow \infty} \phi\left(u, u_{n}\right)=\lim _{n \rightarrow \infty} \phi\left(u, \tilde{u}_{n}\right) .
$$

Indeed, from (4.6e) we get

$$
\phi\left(u, x_{n+1}\right)-\tilde{\alpha}_{n} \phi\left(u, x_{0}\right) \leq \phi\left(u, \tilde{x}_{n}\right) \leq \phi\left(u, x_{n}\right)+\alpha_{n} \phi\left(u, x_{0}\right),
$$

which, together with $\lim _{n \rightarrow \infty} \alpha_{n}=\lim _{n \rightarrow \infty} \tilde{\alpha}_{n}=0$, yields that

$$
\lim _{n \rightarrow \infty} \phi\left(u, \tilde{x}_{n}\right)=\lim _{n \rightarrow \infty} \phi\left(u, x_{n}\right)
$$


From (4.6d) it follows that

$$
\phi\left(u, x_{n+1}\right)-\tilde{\alpha}_{n} \phi\left(u, x_{0}\right) \leq \phi\left(u, \tilde{y}_{n}\right) \leq \phi\left(u, \tilde{x}_{n}\right),
$$

which, together with $\lim _{n \rightarrow \infty} \phi\left(u, \tilde{x}_{n}\right)=\lim _{n \rightarrow \infty} \phi\left(u, x_{n}\right)$, yields that

$$
\lim _{n \rightarrow \infty} \phi\left(u, \tilde{y}_{n}\right)=\lim _{n \rightarrow \infty} \phi\left(u, x_{n}\right)
$$

From (4.6c) it follows that

$$
\phi\left(u, \tilde{x}_{n}\right)-\alpha_{n} \phi\left(u, x_{0}\right) \leq \phi\left(u, y_{n}\right) \leq \phi\left(u, x_{n}\right),
$$

which, together with $\lim _{n \rightarrow \infty} \phi\left(u, \tilde{x}_{n}\right)=\lim _{n \rightarrow \infty} \phi\left(u, x_{n}\right)$, yields that

$$
\lim _{n \rightarrow \infty} \phi\left(u, y_{n}\right)=\lim _{n \rightarrow \infty} \phi\left(u, x_{n}\right)
$$

From (4.6c) it follows that

$$
\phi\left(u, \tilde{y}_{n}\right) \leq \phi\left(u, \tilde{u}_{n}\right) \leq \phi\left(u, \tilde{x}_{n}\right)
$$

which together with

$$
\lim _{n \rightarrow \infty} \phi\left(u, \tilde{x}_{n}\right)=\lim _{n \rightarrow \infty} \phi\left(u, \tilde{y}_{n}\right)=\lim _{n \rightarrow \infty} \phi\left(u, x_{n}\right),
$$

yields that

$$
\lim _{n \rightarrow \infty} \phi\left(u, \tilde{u}_{n}\right)=\lim _{n \rightarrow \infty} \phi\left(u, x_{n}\right)
$$

From (4.6a) it follows that

$$
\phi\left(u, y_{n}\right) \leq \phi\left(u, u_{n}\right) \leq \phi\left(u, x_{n}\right)
$$

which, together with $\lim _{n \rightarrow \infty} \phi\left(u, y_{n}\right)=\lim _{n \rightarrow \infty} \phi\left(u, x_{n}\right)$, yields that

$$
\lim _{n \rightarrow \infty} \phi\left(u, u_{n}\right)=\lim _{n \rightarrow \infty} \phi\left(u, x_{n}\right)
$$

On the other hand, let us show that

$$
\lim _{n \rightarrow \infty}\left\|x_{n}-\tilde{x}_{n}\right\|=0
$$


Indeed, let $s=\sup \left\{\left\|x_{n}\right\|,\left\|u_{n}\right\|,\left\|\tilde{x}_{n}\right\|,\left\|\tilde{u}_{n}\right\|: n \geq 0\right\}$. From Lemma 2.7, there exists a continuous, strictly increasing, and convex function $g_{1}$ with $g_{1}(0)=0$ such that

$$
g_{1}(\|x-y\|) \leq \phi(x, y), \quad \forall x, y \in B_{s} .
$$

Since $u_{n}=K_{r_{n}} x_{n}$ and $\tilde{u}_{n}=K_{r_{n}} \tilde{x}_{n}$, we deduce from Proposition 2.11 that for $u \in \Omega$,

$$
\begin{aligned}
& g_{1}\left(\left\|u_{n}-x_{n}\right\|\right) \leq \phi\left(u_{n}, x_{n}\right) \leq \phi\left(u, x_{n}\right)-\phi\left(u, u_{n}\right), \\
& g_{1}\left(\left\|\tilde{u}_{n}-\tilde{x}_{n}\right\|\right) \leq \phi\left(\tilde{u}_{n}, \tilde{x}_{n}\right) \leq \phi\left(u, \tilde{x}_{n}\right)-\phi\left(u, \tilde{u}_{n}\right) .
\end{aligned}
$$

This implies that

$$
\lim _{n \rightarrow \infty} g_{1}\left(\left\|u_{n}-x_{n}\right\|\right)=\lim _{n \rightarrow \infty} g_{1}\left(\left\|\tilde{u}_{n}-\tilde{x}_{n}\right\|\right)=0
$$

Since $J$ is uniformly norm-to-norm continuous on bounded subsets of $X$, from the properties of $g_{1}$, we obtain

$$
\begin{aligned}
& \lim _{n \rightarrow \infty}\left\|u_{n}-x_{n}\right\|=\lim _{n \rightarrow \infty}\left\|J u_{n}-J x_{n}\right\|=0, \\
& \lim _{n \rightarrow \infty}\left\|\tilde{u}_{n}-\tilde{x}_{n}\right\|=\lim _{n \rightarrow \infty}\left\|J \tilde{u}_{n}-J \tilde{x}_{n}\right\|=0 .
\end{aligned}
$$

Note that

$$
\begin{aligned}
\phi\left(x_{n}, u_{n}\right)-\phi\left(u_{n}, x_{n}\right)= & \left\|x_{n}\right\|^{2}-2\left\langle x_{n}, J u_{n}\right\rangle+\left\|u_{n}\right\|^{2}-\left[\left\|x_{n}\right\|^{2}-2\left\langle u_{n}, J x_{n}\right\rangle+\left\|u_{n}\right\|^{2}\right] \\
= & -2\left\langle x_{n}, J u_{n}\right\rangle+2\left\langle u_{n}, J x_{n}\right\rangle \\
= & 2\left\langle x_{n}, J x_{n}-J u_{n}\right\rangle+2\left\langle u_{n}-x_{n}, J x_{n}\right\rangle \\
\leq & 2\left\|x_{n}\right\|\left\|J x_{n}-J u_{n}\right\|+2\left\|u_{n}-x_{n}\right\|\left\|x_{n}\right\|, \\
\phi\left(x_{n}, J_{r_{n}} u_{n}\right)= & \left\|x_{n}\right\|^{2}-2\left\langle x_{n}, J J_{r_{n}} u_{n}\right\rangle+\left\|J_{r_{n}} u_{n}\right\|^{2} \\
= & \left\|x_{n}\right\|^{2}-\left\|x_{n}\right\|^{2}+\left\|J_{r_{n}} u_{n}\right\|^{2}-\left\|x_{n}\right\|^{2}+2\left\langle x_{n}, J x_{n}-J J_{r_{n}} u_{n}\right\rangle \\
= & \left(\left\|J_{r_{n}} u_{n}\right\|-\left\|x_{n}\right\|\right)\left(\left\|J_{r_{n}} u_{n}\right\|+\left\|x_{n}\right\|\right)+2\left\langle x_{n}, J x_{n}-J J_{r_{n}} u_{n}\right\rangle \\
\leq & \left\|J_{r_{n}} u_{n}-x_{n}\right\|\left(\left\|J_{r_{n}} u_{n}\right\|+\left\|x_{n}\right\|\right)+2\left\|x_{n}\right\|\left\|J x_{n}-J J_{r_{n}} u_{n}\right\| \\
= & \left\|J_{r_{n}} u_{n}-u_{n}+u_{n}-x_{n}\right\|\left(\left\|J_{r_{n}} u_{n}\right\|+\left\|x_{n}\right\|\right) \\
& +2\left\|x_{n}\right\|\left\|J x_{n}-J u_{n}+J u_{n}-J J_{r_{n}} u_{n}\right\| \\
\leq & \left(\left\|J_{r_{n}} u_{n}-u_{n}\right\|+\left\|u_{n}-x_{n}\right\|\right)\left(\left\|J_{r_{n}} u_{n}\right\|+\left\|x_{n}\right\|\right) \\
& +2\left\|x_{n}\right\|\left(\left\|J x_{n}-J u_{n}\right\|+\left\|J u_{n}-J J_{r_{n}} u_{n}\right\|\right) .
\end{aligned}
$$

Since $\phi\left(u_{n}, x_{n}\right) \rightarrow 0$, it follows from (4.24) and (4.41) that $\phi\left(x_{n}, u_{n}\right) \rightarrow 0$ and $\phi\left(x_{n}, J_{r_{n}} u_{n}\right) \rightarrow$ 0 . 
Also, observe that

$$
\begin{aligned}
\phi\left(x_{n}, y_{n}\right) & =\phi\left(x_{n}, J^{-1}\left(\beta_{n} J u_{n}+\left(1-\beta_{n}\right) J J_{r_{n}} u_{n}\right)\right) \\
& =\left\|x_{n}\right\|^{2}-2\left\langle x_{n}, \beta_{n} J u_{n}+\left(1-\beta_{n}\right) J J_{r_{n}} u_{n}\right\rangle+\left\|\beta_{n} J u_{n}+\left(1-\beta_{n}\right) J J_{r_{n}} u_{n}\right\|^{2} \\
& \leq\left\|x_{n}\right\|^{2}-2 \beta_{n}\left\langle x_{n}, J u_{n}\right\rangle-2\left(1-\beta_{n}\right)\left\langle x_{n}, J J_{r_{n}} u_{n}\right\rangle+\beta_{n}\left\|u_{n}\right\|^{2}+\left(1-\beta_{n}\right)\left\|J_{r_{n}} u_{n}\right\|^{2} \\
& =\beta_{n} \phi\left(x_{n}, u_{n}\right)+\left(1-\beta_{n}\right) \phi\left(x_{n}, J_{r_{n}} u_{n}\right) \\
& \leq \phi\left(x_{n}, u_{n}\right)+\phi\left(x_{n}, J_{r_{n}} u_{n}\right),
\end{aligned}
$$

and hence

$$
\begin{aligned}
\phi\left(x_{n}, \tilde{x}_{n}\right) & =\phi\left(x_{n}, J^{-1}\left(\alpha_{n} J x_{0}+\left(1-\alpha_{n}\right) J y_{n}\right)\right) \\
& =\left\|x_{n}\right\|^{2}-2\left\langle x_{n}, \alpha_{n} J x_{0}+\left(1-\alpha_{n}\right) J y_{n}\right\rangle+\left\|\alpha_{n} J x_{0}+\left(1-\alpha_{n}\right) J y_{n}\right\|^{2} \\
& \leq\left\|x_{n}\right\|^{2}-2 \alpha_{n}\left\langle x_{n}, J x_{0}\right\rangle-2\left(1-\alpha_{n}\right)\left\langle x_{n}, J y_{n}\right\rangle+\alpha_{n}\left\|x_{0}\right\|^{2}+\left(1-\alpha_{n}\right)\left\|y_{n}\right\|^{2} \\
& =\alpha_{n} \phi\left(x_{n}, x_{0}\right)+\left(1-\alpha_{n}\right) \phi\left(x_{n}, y_{n}\right) \\
& \leq \alpha_{n} \phi\left(x_{n}, x_{0}\right)+\phi\left(x_{n}, y_{n}\right) \\
& \leq \alpha_{n} \phi\left(x_{n}, x_{0}\right)+\phi\left(x_{n}, u_{n}\right)+\phi\left(x_{n}, J_{r_{n}} u_{n}\right) .
\end{aligned}
$$

Thus, from $\alpha_{n} \rightarrow 0, \phi\left(x_{n}, u_{n}\right) \rightarrow 0$, and $\phi\left(x_{n}, J_{r_{n}} u_{n}\right) \rightarrow 0$, it follows that $\phi\left(x_{n}, \tilde{x}_{n}\right) \rightarrow 0$. In terms of Lemma 2.2, we derive $\left\|x_{n}-\tilde{x}_{n}\right\| \rightarrow 0$.

Next, let us show that $x_{n} \rightarrow z$, where $z=\lim _{n \rightarrow \infty} \Pi_{T^{-1} 0 \cap \tilde{T}^{-1} 0 \cap E P} x_{n}$.

Indeed, since $\left\{x_{n}\right\}$ is bounded, there exists a subsequence $\left\{x_{n_{k}}\right\}$ of $\left\{x_{n}\right\}$ such that $x_{n_{k}} \rightarrow$ $\hat{x} \in C$. Hence it follows from (4.24), (4.41), and $\left\|x_{n}-\tilde{x}_{n}\right\| \rightarrow 0$ that $\left\{u_{n_{k}}\right\},\left\{\tilde{u}_{n_{k}}\right\},\left\{J_{r_{n_{k}}} u_{n_{k}}\right\}$ and $\tilde{J}_{r_{k}} \tilde{u}_{n_{k}}$ converge weakly to the same point $\widehat{x}$. Furthermore, from $\lim \inf _{n \rightarrow \infty} r_{n}>0$ and (4.24), we have that

$$
\begin{aligned}
& \lim _{n \rightarrow \infty}\left\|A_{r_{n}} u_{n}\right\|=\lim _{n \rightarrow \infty} \frac{1}{r_{n}}\left\|J u_{n}-J J_{r_{n}} u_{n}\right\|=0, \\
& \lim _{n \rightarrow \infty}\left\|\widetilde{A}_{r_{n}} \tilde{u}_{n}\right\|=\lim _{n \rightarrow \infty} \frac{1}{r_{n}}\left\|J \tilde{u}_{n}-J \widetilde{J}_{r_{n}} \tilde{u}_{n}\right\|=0 .
\end{aligned}
$$

If $z^{*} \in T z$ and $\widetilde{z}^{*} \in \widetilde{T} \widetilde{z}$, then it follows from (2.17) and the monotonicity of the operators $T, \tilde{T}$ that for all $k \geq 1$

$$
\left\langle z-J_{r_{n_{k}}} u_{n_{k}}, z^{*}-A_{r_{n_{k}}} u_{n_{k}}\right\rangle \geq 0, \quad\left\langle\tilde{z}-\tilde{J}_{r_{n_{k}}} \tilde{u}_{n_{k}}, \widetilde{z}^{*}-\tilde{A}_{r_{n_{k}}} \tilde{u}_{n_{k}}\right\rangle \geq 0 .
$$

Letting $k \rightarrow \infty$, we obtain that

$$
\left\langle z-\widehat{x}, z^{*}\right\rangle \geq 0, \quad\left\langle\widetilde{z}-\widehat{x}, \widetilde{z}^{*}\right\rangle \geq 0 .
$$

Then the maximality of the operators $T, \widetilde{T}$ implies that $\widehat{x} \in T^{-1} 0 \cap \widetilde{T}^{-1} 0$. 
Now, by the definition of $u_{n}:=K_{r_{n}} x_{n}$, we have

$$
F\left(u_{n}, y\right)+\frac{1}{r_{n}}\left\langle y-u_{n}, J u_{n}-J x_{n}\right\rangle \geq 0, \quad \forall y \in C,
$$

where $F(x, y)=f(x, y)+\langle A x, y-x\rangle$. Replacing $n$ by $n_{k}$, we have from (A2) that

$$
\frac{1}{r_{n_{k}}}\left\langle y-u_{n_{k}}, J u_{n_{k}}-J x_{n_{k}}\right\rangle \geq-F\left(u_{n_{k}}, y\right) \geq F\left(y, u_{n_{k}}\right), \quad \forall y \in C .
$$

Since $y \mapsto F(x, y)$ is convex and lower semicontinuous, it is also weakly lower semicontinuous. Letting $n_{k} \rightarrow \infty$ in the last inequality, from (4.41) and (A4), we have

$$
F(y, \widehat{x}) \leq 0, \quad \forall y \in C
$$

For $t$, with $0<t \leq 1$, and $y \in C$, let $y_{t}=t y+(1-t) \widehat{x}$. Since $y \in C$ and $\widehat{x} \in C$, then $y_{t} \in C$ and hence $F\left(y_{t}, \widehat{x}\right) \leq 0$. So, from (A1), we have

$$
0=F\left(y_{t}, y_{t}\right) \leq t F\left(y_{t}, y\right)+(1-t) F\left(y_{t}, \widehat{x}\right) \leq t F\left(y_{t}, y\right)
$$

Dividing by $t$, we get $F\left(y_{t}, y\right) \geq 0, \forall y \in C$. Letting $t \downarrow 0$, from (A3) it follows that $F(\widehat{x}, y) \geq$ $0, \forall y \in C$. So, $\widehat{x} \in E P$. Therefore, $\widehat{x} \in \Omega$. Let $z_{n}=\Pi_{\Omega} x_{n}$. From Lemma 2.3 and $\widehat{x} \in \Omega$, we get

$$
\left\langle z_{n_{k}}-\widehat{x}, J x_{n_{k}}-J z_{n_{k}}\right\rangle \geq 0
$$

From Proposition 4.1, we also know that $z_{n} \rightarrow z \in \Omega$. Note that $x_{n_{k}} \rightarrow \widehat{x}$. Since $J$ is weakly sequentially continuous, then $\langle z-\widehat{x}, J \widehat{x}-J z\rangle \geq 0$ as $k \rightarrow \infty$. In addition, taking into account the monotonicity of $J$, we conclude that $\langle z-\widehat{x}, J \widehat{x}-J z\rangle \leq 0$. Hence

$$
\langle z-\widehat{x}, J \widehat{x}-J z\rangle=0
$$

From the strict convexity of $X$, it follows that $z=\hat{x}$. Therefore, $x_{n} \rightarrow \widehat{x}$, where $\hat{x}=$ $\lim _{n \rightarrow \infty} \prod_{T^{-1} 0 \cap \widetilde{T}^{-1} 0 \cap E P} x_{n}$. This completes the proof.

Remark 4.3. Compared with the algorithm of Theorem 1.2, the above algorithm (4.1) can be applied to find an element of $T^{-1} 0 \cap \widetilde{T}^{-1} 0 \cap E P$. But, the algorithm of Theorem 1.2 cannot be applied. Therefore, algorithm (4.1) develops and improves the algorithm of Theorem 1.2.

\section{Acknowledgments}

In this research, the first author was partially supported by the Leading Academic Discipline Project of Shanghai Normal University (DZL707), Innovation Program of Shanghai Municipal Education Commission Grant (09ZZ133), National Science Foundation of China (10771141), Ph.D. Program Foundation of Ministry of Education of China (20070270004), 
Science and Technology Commission of Shanghai Municipality Grant (075105118), and Shanghai Leading Academic Discipline Project (S30405). The Fourth author was partially supported by a grant NSC 98-2115-M-110-001.

\section{References}

[1] S.-S. Zhang, "Shrinking projection method for solving generalized equilibrium problem, variational inequality and common fixed point in Banach spaces with applications," to appear in Science in China Series $A$.

[2] L.-C. Zeng, Q. H. Ansari, and J.-C. Yao, "Viscosity approximation methods for generalized equilibrium problems and fixed point problems," Journal of Global Optimization, vol. 43, no. 4, pp. 487-502, 2009.

[3] L.-C. Zeng, C. Lee, and J.-C. Yao, "Strong weak convergence theorems of implicit hybrid steepestdescent methods for variational inequalities," Taiwanese Journal of Mathematics, vol. 12, no. 1, pp. 227244, 2008.

[4] L.-C. Zeng, S.-Y. Wu, and J.-C. Yao, "Generalized KKM theorem with applications to generalized minimax inequalities and generalized equilibrium problems," Taiwanese Journal of Mathematics, vol. 10, no. 6, pp. 1497-1514, 2006.

[5] J.-W. Peng and J.-C. Yao, "A new hybrid-extragradient method for generalized mixed equilibrium problems, fixed point problems and variational inequality problems," Taiwanese Journal of Mathematics, vol. 12, no. 6, pp. 1401-1432, 2008.

[6] J.-W. Peng and J.-C. Yao, "Some new extragradient-like methods forgeneralized equilibrium problems, fixed points problems and variational inequality problems," to appear in Optimization Methods and Software.

[7] J.-W. Peng and J.-C. Yao, "Strong convergence theorems of iterative scheme based on the extragradient method for mixed equilibrium problems and fixed point problems," Mathematical and Computer Modelling, vol. 49, no. 9-10, pp. 1816-1828, 2009.

[8] J.-W. Peng and J.-C. Yao, "Some new iterative algorithms for generalized mixed equilibrium problems with strict pseudo-contractions and monotone mappings," Taiwanese Journal of Mathematics, vol. 13, no. 5, pp. 1537-1582, 2009.

[9] J.-W. Peng and J.-C. Yao, "Ishikawa iterative algorithms for a generalized equilibrium problem and fixed point problems of a pseudo-contraction mapping," to appear in Journal of Global Optimization.

[10] S. Schaible, J.-C. Yao, and L.-C. Zeng, "A proximal method for pseudomonotone type variational-like inequalities," Taiwanese Journal of Mathematics, vol. 10, no. 2, pp. 497-513, 2006.

[11] L. C. Zeng, L. J. Lin, and J. C. Yao, "Auxiliary problem method for mixed variational-like inequalities," Taiwanese Journal of Mathematics, vol. 10, no. 2, pp. 515-529, 2006.

[12] L.-C. Zeng and J.-C. Yao, "Strong convergence theorem by an extragradient method for fixed point problems and variational inequality problems," Taiwanese Journal of Mathematics, vol. 10, no. 5, pp. 1293-1303, 2006.

[13] S. Takahashi and W. Takahashi, "Viscosity approximation methods for equilibrium problems and fixed point problems in Hilbert spaces," Journal of Mathematical Analysis and Applications, vol. 331, no. 1, pp. 506-515, 2007.

[14] E. Blum and W. Oettli, "From optimization and variational inequalities to equilibrium problems," The Mathematics Student, vol. 63, no. 1-4, pp. 123-145, 1994.

[15] L.-C. Zeng and J.-C. Yao, "A hybrid iterative scheme for mixed equilibrium problems and fixed point problems," Journal of Computational and Applied Mathematics, vol. 214, no. 1, pp. 186-201, 2008.

[16] L.-C. Zeng and J.-C. Yao, "Hybrid viscosity approximation schemes for equilibrium problems and fixed point problems of infinitely many nonexpansive mappings," Applied Mathematics and Computation, vol. 198, no. 2, pp. 729-741, 2008.

[17] L. C. Zeng, G. Mastroeni, and J. C. Yao, "Hybrid proximal-point methods for common solutions of equilibrium problems and zeros of maximal monotone operators," Journal of Optimization Theory and Applications, vol. 142, no. 3, pp. 431-449, 2009.

[18] W. Takahashi and K. Zembayashi, "Strong and weak convergence theorems for equilibrium problems and relatively nonexpansive mappings in Banach spaces," Nonlinear Analysis: Theory, Methods $\mathcal{E}$ Applications, vol. 70, no. 1, pp. 45-57, 2009.

[19] B. Martinet, "Régularisation d'inéquations variationnelles par approximations successives," Revue Française d'Informatique et de Recherche Opérationnelle, vol. 4, pp. 154-158, 1970. 
[20] R. T. Rockafellar, "Monotone operators and the proximal point algorithm," SIAM Journal on Control and Optimization, vol. 14, no. 5, pp. 877-898, 1976.

[21] O. Güler, "On the convergence of the proximal point algorithm for convex minimization," SIAM Journal on Control and Optimization, vol. 29, no. 2, pp. 403-419, 1991.

[22] S. Kamimura, F. Kohsaka, and W. Takahashi, "Weak and strong convergence theorems for maximal monotone operators in a Banach space," Set-Valued Analysis, vol. 12, no. 4, pp. 417-429, 2004.

[23] F. Kohsaka and W. Takahashi, "Strong convergence of an iterative sequence for maximal monotone operators in a Banach space," Abstract and Applied Analysis, no. 3, pp. 239-249, 2004.

[24] L. Li and W. Song, "Modified proximal-point algorithm for maximal monotone operators in Banach spaces," Journal of Optimization Theory and Applications, vol. 138, no. 1, pp. 45-64, 2008.

[25] L.-C. Zeng and J.-C. Yao, "An inexact proximal-type algorithm in Banach spaces," Journal of Optimization Theory and Applications, vol. 135, no. 1, pp. 145-161, 2007.

[26] Y. I. Alber, "Metric and generalized projection operators in Banach spaces: properties and applications," in Theory and Applications of Nonlinear Operators of Accretive and Monotone Type, A. G. Kartsatos, Ed., vol. 178 of Lecture Notes in Pure and Applied Mathematics, pp. 15-50, Marcel Dekker, New York, NY, USA, 1996.

[27] Y. I. Alber and S. Guerre-Delabriere, "On the projection methods for fixed point problems," Analysis, vol. 21, no. 1, pp. 17-39, 2001.

[28] S. Reich, "A weak convergence theorem for the alternating method with Bregman distances," in Theory and Applications of Nonlinear Operators of Accretive and Monotone Type, A. G. Kartsatos, Ed., vol. 178 of Lecture Notes in Pure and Applied Mathematics, pp. 313-318, Marcel Dekker, New York, NY, USA, 1996.

[29] S. Matsushita and W. Takahashi, "Weak and strong convergence theorems for relatively nonexpansive mappings in Banach spaces," Fixed Point Theory and Applications, vol. 2004, no. 1, pp. 37-47, 2004.

[30] S. Matsushita and W. Takahashi, "A strong convergence theorem for relatively nonexpansive mappings in a Banach space," Journal of Approximation Theory, vol. 134, no. 2, pp. 257-266, 2005.

[31] I. Cioranescu, Geometry of Banach Spaces, Duality Mappings and Nonlinear Problems, vol. 62 of Mathematics and Its Applications, Kluwer Academic Publishers, Dordrecht, The Netherlands, 1990.

[32] S. Kamimura and W. Takahashi, "Strong convergence of a proximal-type algorithm in a Banach space," SIAM Journal on Optimization, vol. 13, no. 3, pp. 938-945, 2002.

[33] R. T. Rockafellar, "On the maximality of sums of nonlinear monotone operators," Transactions of the American Mathematical Society, vol. 149, pp. 75-88, 1970.

[34] H. K. Xu, "Inequalities in Banach spaces with applications," Nonlinear Analysis: Theory, Methods E Applications, vol. 16, no. 12, pp. 1127-1138, 1991.

[35] P. L. Combettes and S. A. Hirstoaga, "Equilibrium programming in Hilbert spaces," Journal of Nonlinear and Convex Analysis, vol. 6, no. 1, pp. 117-136, 2005.

[36] K.-K. Tan and H. K. Xu, "Approximating fixed points of nonexpansive mappings by the Ishikawa iteration process," Journal of Mathematical Analysis and Applications, vol. 178, no. 2, pp. 301-308, 1993. 